\title{
Improved Methods For Generating Quasi-Gray Codes
}

\author{
By \\ Dana Jansens

\begin{abstract}
A thesis submitted to
the Faculty of Graduate Studies and Research

in partial fulfilment of

the requirements for the degree of

Master of Computer Science
\end{abstract} \\ Ottawa-Carleton Institute for Computer Science \\ School of Computer Science \\ Carleton University \\ Ottawa, Ontario
}

April 2010

(C) Copyright

2010, Dana Jansens 


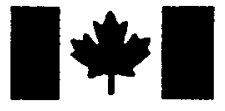

Library and Archives

Canada

Published Heritage

Branch

395 Wellington Street

Ottawa ON K1A ON4

Canada
Bibliotheqque et

Archives Canada

Direction du

Patrimoine de l'édition

395, rue Wellington

Ottawa ON K1A ON4

Canada
Your file Votre refference

ISBN: 978-0-494-68632-4

Our file Notre référence

ISBN: 978-0-494-68632-4
NOTICE:

The author has granted a nonexclusive license allowing Library and Archives Canada to reproduce, publish, archive, preserve, conserve, communicate to the public by telecommunication or on the Internet, loan, distribute and sell theses worldwide, for commercial or noncommercial purposes, in microform, paper, electronic and/or any other formats.

The author retains copyright ownership and moral rights in this thesis. Neither the thesis nor substantial extracts from it may be printed or otherwise reproduced without the author's permission.
AVIS:

L'auteur a accordé une licence non exclusive permettant à la Bibliothèque et Archives Canada de reproduire, publier, archiver, sauvegarder, conserver, transmettre au public par télécommunication ou par l'Internet, prêter, distribuer et vendre des thèses partout dans le monde, à des fins commerciales ou autres, sur support microforme, papier, électronique et/ou autres formats.

L'auteur conserve la propriété du droit d'auteur et des droits moraux qui protège cette thèse. $\mathrm{Ni}$ la thèse ni des extraits substantiels de celle-ci ne doivent être imprimés ou autrement reproduits sans son autorisation.
In compliance with the Canadian Privacy Act some supporting forms may have been removed from this thesis.

While these forms may be included in the document page count, their removal does not represent any loss of content from the thesis.
Conformément à la loi canadienne sur la protection de la vie privée, quelques formulaires secondaires ont été enlevés de cette thèse.

Bien que ces formulaires aient inclus dans la pagination, il n'y aura aucun contenu manquant.

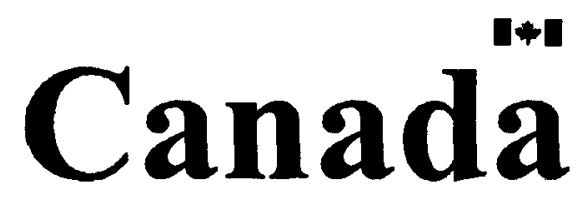




\section{Abstract}

Consider a sequence of bit strings of length $d$, such that each string differs from the next in a constant number of bits. We call this sequence a quasi-Gray code. We examine the problem of efficiently generating such codes, by considering the number of bits read and written at each generating step, the average number of bits read while generating the entire code, and the number of strings generated in the code. Our results give a trade-off between these constraints, and present algorithms that do less work on average than previous results, and that increase the number of bit strings generated. 


\section{Acknowledgements}

I must say a big thank you to my supervisors Anil Maheshwari and Michiel Smid, each of whom must have read parts of this thesis at least ten times by now. Their support and input has helped a great deal in my success in this degree program. I also want to thank Pat Morin, who worked with me on many parts of this thesis and provided a lot of feedback on the small details of my writing, even though he was not supervising me and does not get official credit for it. I wish to thank Prosenjit Bose and Paz Carmi as well for input on these problems, and Lucia Moura and Brett Stevens for being on my thesis defence committee. I must also send a thank you to Anil Somayaji, who invited me to join in writing a paper during my third year of undergrad studies. I never anticipated at the time that it would lead to me attending grad school and eventually writing an entire thesis. My inspiration and ability to write this thesis can be attributed in no small way to role models such as these.

I want to give a big shout-out to the entire Carleton computational geometry lab crew, with who I eat lunch every day, who listen to my research presentations, discuss research ideas with me, and who make me feel welcome and a legitimate part of the group. Their presence has been vital to me, and I know I would have struggled much more in this degree without them. I especially want to thank Prosenjit Bose as he goes out of his way to keep the group together and to make the space welcoming to us all.

I have been fortunate to find a lot of support and encouragement in my life, without which I know this accomplishment would not be possible. Djamila Ibrahim, Heather Larke, Larry and Sue Larke, and Lisa Cooper have all been there to support me, believe in me, push me, and catch me, and I am very grateful for it all. 
I will continue to push myself on to my next, bigger, challenge thanks to all of these people. 


\section{Contents}

Abstract $\quad$ ii

Acknowledgements $\quad$ iii

1 Introduction 1

1.1 Problem Statement . . . . . . . . . . . . . . . . . . . 1

1.2 Definitions . . . . . . . . . . . . . . . . 3

1.3 Results Summary . . . . . . . . . . . . . . . . 3

1.4 Organization of the thesis ................ 6

2 Previous work $\quad 7$

2.1 Gray codes . . . . . . . . . . . . . . . . 7

2.2 Quasi-Gray codes . . . . . . . . . . . . . . 8

2.3 Upper and lower bounds . . . . . . . . . . . . . . 11

3 Decision Assignment Trees 13

3.1 The Decision Assignment Tree Model . . . . . . . . . . . . . . . 13

3.2 Assembling DATs . . . . . . . . . . . . . . . . . 15

3.3 Generating the BRGC . . . . . . . . . . . . 16

4 Efficient generation of quasi-Gray codes $\quad 18$

4.1 Recursive Partition Gray Code (RPGC) . . . . . . . . . . . . 18

4.2 Composite code construction . . . . . . . . . . . . . . . 37

4.3 RPGC-Composite Code . . . . . . . . . . . . . . . 40 
4.4 Reading a constant average number of bits . . . . . . . . . . . 43

4.5 Lazy counters . . . . . . . . . . . . . . . . . . . 45

4.5 .1 LazyIncrement . . . . . . . . . . . . . 45

4.5 .2 SpinIncrement . . . . . . . . . . . . . 47

4.5 .3 DoubleSpinIncrement . . . . . . . . . . . . 49

4.5 .4 WineIncrement ................. 51

5 Conclusion $\quad 60$

5.1 Summary ......................... 60

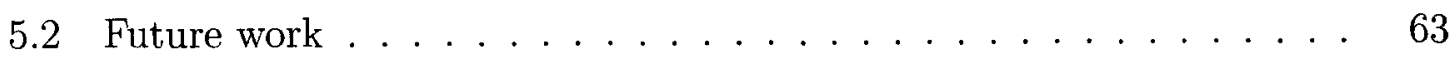

$\begin{array}{lr}\text { Bibliography } & 64\end{array}$ 


\section{List of Figures}

2.1 The standard Binary Reflected Gray Code for 1,2 , and 3 dimensions 8

3.1 A DAT that generates the Binary Reflected Gray Code on three bits. The bits are labelled from 0 as the right-most to 2 as the left-most bit. The label in each internal node specifies which bit is being read. Control moves to the left child or right child if the bit is equal to 0 or 1 , respectively. The rules in the leaf nodes set a bit $i$ to a new value,

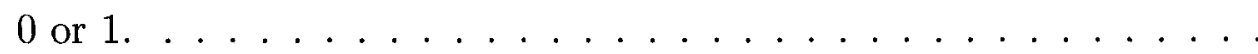

4.1 Conceptualization of the Recursive Partition Gray Code. $A$ moves clockwise while $A \neq B$, at which point $B$ moves counter-clockwise. . .

4.2 A conceptualization of the Composite code. $B$ moves clockwise until it reaches 0 , at which point $A$ moves clockwise one position also. . . .

5.1 Rules for traversing a hypercube of dimension $d=3$ that require reading $d-1$ bits. The rules are directed dashed lines, each of which shows the bit that would be changed when the code's current bit string is equal to the label of the rule's source vertex in the hypercube. A dark line connects two states that differ only in the bit not read for the two paired rules adjacent to it. . . . . . . . . . . . . 


\section{Chapter 1}

\section{Introduction}

\section{$1.1 \quad$ Problem Statement}

We are interested in efficiently generating a sequence of bit strings. The class of bit strings we wish to generate are cyclic quasi-Gray codes. A Gray code [Gra53] is a sequence of bit strings, such that any two consecutive strings differ in exactly one bit. We use the term quasi-Gray code [Fre78] to refer to a sequence of bit strings where any two consecutive strings differ in at most $c$ bits, where $c$ is a constant defined for the code. A Gray code (quasi-Gray code) is called cyclic if the first and last generated bit strings also differ in at most 1 bit ( $c$ bits).

We say a bit string that contains $d$ bits has dimension $d$, and are interested in efficient algorithms to generate a sequence of bit strings that form a quasi-Gray code of dimension $d$. After generating a bit string, we say the algorithm's data structure corresponds exactly to the generated bit string, and it's state is the bit string itself. In this way, we restrict an algorithm's data structure to using exactly $d$ bits. At each step, the input to the algorithm will be a bit string, which is the algorithm's current state. The output will be a new bit string that corresponds to the next state of the algorithm's data structure.

The number of consecutive unique bit strings generated is equal to the number of consecutive unique states for the generating data structure, and we call this value $L$, the length of the generated code. Clearly $L \leq 2^{d}$. We define the space efficiency of 
an algorithm as the ratio $L / 2^{d}$, that is, the fraction of bit strings generated out of all possible bit strings given the dimension of the strings. When the space efficiency is 1 , we call the data structure space-optimal, as it generates all possible bit strings. When $L<2^{d}$, the structure is non-space-optimal; as we will see, this allows the time required to generate each consecutive bit string to be improved.

Each generating step takes as input the output of the previous generating step, which is a bit string in the quasi-Gray code. The average number of bits read is defined to be the ratio of the total number of bits read, to the length of the code, when generating one iteration of the entire quasi-Gray code.

Our goal is to study and improve efficiency of algorithms for generating quasi-Gray codes in the following ways.

1. Worst-Case read: We would like to know how many bits the algorithm must read in the worst case in order to make the appropriate changes in the input string and generate the next bit string in the code, and find ways to reduce this when possible.

2. Worst-case write: We would like to know how many bits must change in the worst case to reach the successor string in the code, and keep this to 1 when possible while improving upon other metrics.

3. Average number of bits read: We would like to reduce the average number of bits read at each generating step while maintaining other metrics.

4. Space efficiency: We would like our algorithms to be as space efficient as possible, ideally generating as many bit strings as their dimension allows, with $L=2^{d}$.

Our results give a trade-off between these different goals.

Our decision to limit the algorithm's data structure to exactly $d$ bits differs from previous work, where the data structure could use more bits than the strings it generated [Fre78, RM08]. To compare previous results to our own, we consider the extra bits in their data structure to be a part of their generated bit strings. This gives a more precise view of the space efficiency of an algorithm. 
Each generated bit string of dimension $d$ has a distinct totally ordered rank in the generated code with respect to the initial bit string in the code. For a cyclic code, the initial bit string can be chosen arbitrarily. We assume the initial bit string to be the bit string of $d$ zeros unless stated otherwise. Given a string of rank $k$ in a code of length $L$, where $0 \leq k<L$, we want to support the following operations:

- next generates the bit string of rank $(k+1) \bmod L$

- previous generates the bit string of $\operatorname{rank}(k-1) \bmod L$

We work within the bit probe model [MP69, RM08], where the performance of an algorithm is measured by counting the average-case and the worst-case number of bits read and written. We examine these values for the process of generating each bit string in a quasi-Gray code. We use the Decision Assignment Tree (DAT) model [Fre78] (which we describe further in Chapter 3) to construct algorithms for generating quasi-Gray codes and describe the algorithms' behaviour, as well as to discuss upper and lower bounds.

\subsection{Definitions}

We use a notation for the iterated logarithm function of the form $\log ^{(c)} n$ where $c$ is a non-negative integer, and is always surrounded by brackets to differentiate it from an exponent. The value of the function is defined as follows. When $c=0, \log ^{(c)} n=$ $n$. If $c>0$, then $\log ^{(c)}(n)=\log ^{(c-1)}(\log (n))$. For example, $\log ^{(2)} n=\log (\log n)$. Throughout, the base of the log function is assumed to be 2 unless stated otherwise.

We define the function $\log ^{*} n$ to be equal to the smallest non-negative value of $c$ such that $\log ^{(c)} n \leq 1$. For example $\log ^{*} 1=0$ and $\log ^{*} 3=2$.

\subsection{Results Summary}

Our results, as well as previous results, are summarized in Table 1.1. 


\begin{tabular}{|c|c|c|c|c|c|c|c|c|c|c|c|c|c|c|}
\hline & 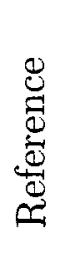 & 总 & 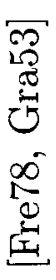 & 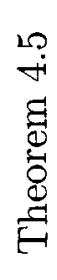 & 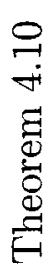 & 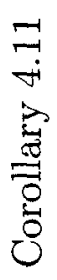 & 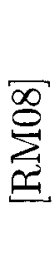 & 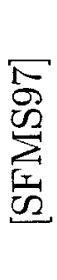 & $\begin{array}{l}\stackrel{8}{8} \\
\stackrel{0}{0}\end{array}$ & 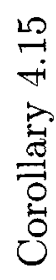 & 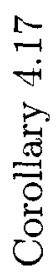 & 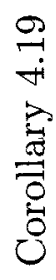 & 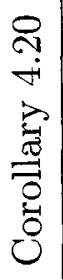 & 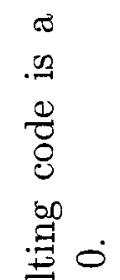 \\
\hline 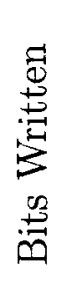 & $\begin{array}{l}0 \\
0 \\
0 \\
0 \\
1 \\
1 \\
0 \\
0 \\
0 \\
0 \\
0\end{array}$ & 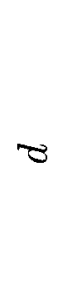 & $\rightarrow$ & -1 & u & 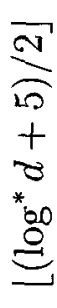 & $\nabla$ & $\begin{array}{l}\overrightarrow{1} \\
+ \\
\text { है } \\
\text { s0 } \\
0\end{array}$ & $\begin{array}{l}\overrightarrow{+} \\
+ \\
\tilde{E} \\
0 \\
0\end{array}$ & $\begin{array}{l}-1 \\
+ \\
5 \\
60 \\
0 \\
ت \\
+ \\
+\end{array}$ & $\infty$ & $\begin{array}{l}\overrightarrow{+} \\
\vec{U}\end{array}$ & $\begin{array}{c}m \\
1 \\
\approx \\
* 00 \\
0\end{array}$ & 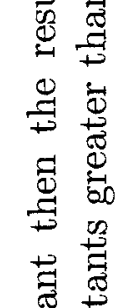 \\
\hline $\mathbb{Z}$ & 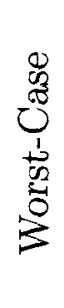 & $\tau$ & $\nabla$ & 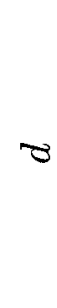 & $\tau$ & $\theta$ & $\begin{array}{l}+ \\
+ \\
0 \\
80 \\
0\end{array}$ & $\begin{array}{l}\overrightarrow{1} \\
+ \\
\varepsilon \\
50 \\
0\end{array}$ & $\begin{array}{l}-1 \\
+ \\
\text { E } \\
\text { D0 } \\
0\end{array}$ & $\begin{array}{l}\overrightarrow{1} \\
+ \\
\tilde{E} \\
60 \\
0 \\
\overrightarrow{-} \\
+ \\
\vec{\sigma}\end{array}$ & 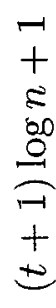 & $\begin{array}{l}-1 \\
+ \\
8 \\
\infty \\
0 \\
\stackrel{-1}{+1} \\
+ \\
\pm\end{array}$ & $\begin{array}{l}\overrightarrow{7} \\
+ \\
8 \\
0 \\
0 \\
\stackrel{1}{7} \\
+ \\
\pm \\
\pm\end{array}$ & 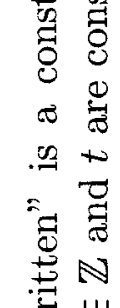 \\
\hline$\stackrel{n}{n}$ & 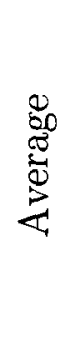 & $\begin{array}{c}7 \\
i \\
i \\
1 \\
N\end{array}$ & $\tau$ & $\begin{array}{l}0 \\
80 \\
0 \\
0\end{array}$ & 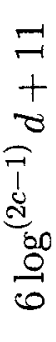 & 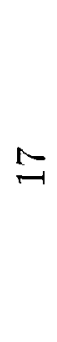 & $\widetilde{\Xi}$ & $\infty$ & 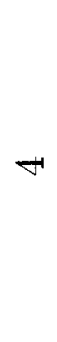 & 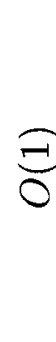 & $\begin{array}{l}\widehat{E} \\
\dot{\infty} \\
\stackrel{0}{ٍ} \\
\stackrel{\omega}{0}\end{array}$ & 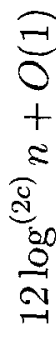 & $\stackrel{\overbrace{}}{\sigma}$ & 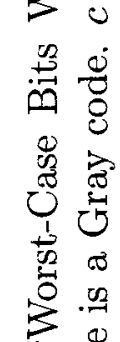 \\
\hline & 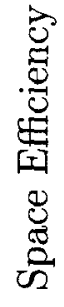 & -1 & $\tau$ & - & $\Gamma$ & $\neg$ & $\underset{-1}{\stackrel{N}{*}}$ & 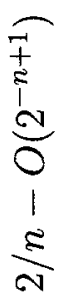 & 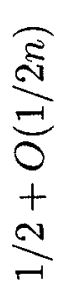 & $\frac{\pi}{1}$ & $\underset{1}{\stackrel{T}{1}}$ & $\overbrace{1}^{\pi}$ & $\underbrace{\vec{j}}_{\overrightarrow{1}}$ & 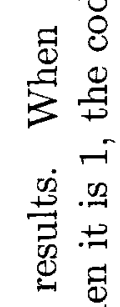 \\
\hline & 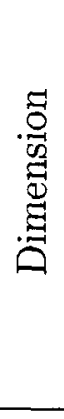 & $\sigma$ & $\sigma$ & $\tau$ & $\sigma$ & $\sigma$ & $\nabla$ & $\begin{array}{l}\vec{E} \\
50 \\
0 \\
+ \\
+ \\
\tilde{R} \\
11 \\
\sigma\end{array}$ & 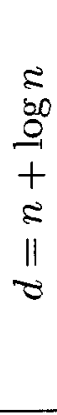 & 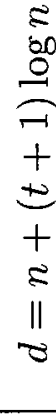 & 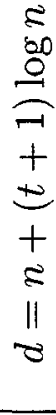 & 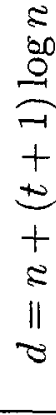 & 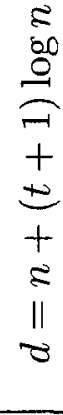 & 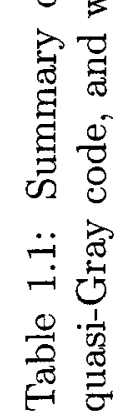 \\
\hline
\end{tabular}


First, we present some space-optimal algorithms. Although our space-optimal algorithms read a small number of bits in the average case, they all read $d$ bits in the worst case.

In Section 4.1, we describe the Recursive Partition Gray Code (RPGC) algorithm, which generates a Gray code of dimension $d$ while reading on average no more than $6 \log d$ bits. This improves the average number of bits read for a space-optimal Gray code from $d$ to $O(\log d)$. In Section 4.2, we use the RPGC to construct a DAT that generates a quasi-Gray code while reducing the average number of bits read. We then apply this technique iteratively in Section 4.3 to create, for any constant $c \geq 1$, a $d$-dimensional DAT that reads worst-case $d$ bits, but reads on average only $6 \log ^{(2 c-1)} d+11$ bits, and writes at most $c$ bits. This lowers the average number of bits read to generate a space-optimal quasi-Gray code from $O(\log d)$ to $O\left(\log ^{(2 c-1)} d\right)$, when $c \geq 2$.

In Section 4.4 we create a $d$-dimensional DAT that reads worst-case all $d$ bits, while reading at most 17 bits on average, and writing at most $\left\lfloor\left(\log ^{*} d+5\right) / 2\right\rfloor$ bits to generate each bit string, while also being space-optimal. This reduces the average number of bits read to $O(1)$ for a space-optimal code, but increases the number of bits written to be slightly more than a constant.

Next, we consider quasi-Gray codes that are not space-optimal, but achieve space efficiency arbitrarily close to 1 , and that read $O(\log d)$ bits in the worst case.

In Section 4.5 we construct a DAT of dimension $d=n+(t+1) \log n$ that reads and writes $(t+1) \log n+1$ bits in the worst case, and has space efficiency $1-O\left(n^{-t}\right)$. This improves the space efficiency dramatically of previous results, when the worst-case number of bits written is $O(\log n)$, from $1 / 2$ to 1 in the limit $n \rightarrow \infty$. By combining a simple Gray code with this result, we are able to produce a DAT of dimension $d=n+(t+1) \log n$ that reads $O(t \log n)$ bits on average and in the worst case, but writes at most 3 bits. This reduces the worst-case number of bits written from $O(\log n)$ to $O(1)$, while the space efficiency remains asymptotically the same.

We then combine results from Section 4.3 to produce a DAT of dimension $d=$ $n+(t+1) \log n$ that reads in the worst case $(t+1) \log n+1$ bits, reads on average $12 \log ^{(2 c)} n+O(1)$ bits, and writes at most $2 c+1$ bits, for any constant $c \geq 1$. This 
improves the average number of bits read to generate a quasi-Gray code with $O(\log d)$ bits read in the worst case. We reduce the average number of bits read from $O(\log d)$ to $O(\log \log d)$ when writing the same number of bits, and when writing a constant number more, the average becomes $O\left(\log ^{(2 c)} d\right)$, while keeping the space efficiency arbitrarily close to 1 . Lastly, we show this DAT can also generate a code while reading a constant number of bits on average, if it writes $\log * n-3$ bits in the worst case.

A summary of the results in this thesis is appearing at SWAT 2010, the 12th Scandinavian Symposium and Workshops on Algorithm Theory.

\subsection{Organization of the thesis}

The remainder of this work will be organized as follows. In Chapter 2, we review related previous work, and discuss its relationship to our own work. In Chapter 3, we present the Decision Assignment Tree model and make some observations about generating quasi-Gray codes within the model. In Chapter 4, we present our results, starting with the Recursive Partition Gray Code, followed by the RPGC-Composite quasi-Gray Code, and finally our Lazy Counters which build up to our WineIncrement counter. We conclude in Chapter 5 , with a summary of our work and discussion of related open problems and future work. 


\section{Chapter 2}

\section{Previous work}

\subsection{Gray codes}

The Gray code was invented by Frank Gray in 1953 [Gra53]. In his patent application, Gray described what we now know as the Binary Reflected Gray Code (BRGC). This code is a sequence of bit strings of dimension $d$, where each successive bit string differs from the previous one in exactly one bit and where all the possible bit strings are present. From this code came the more general term, Gray code, which refers to any sequence of bit strings where successive strings differ in exactly one bit. Furthermore, the concept of a cyclic Gray code was used to describe a Gray code where the first and last bit strings differ in exactly one bit, such as the original BRGC, creating a secondary class of non-cyclic Gray codes.

The BRGC has a structure which can be defined recursively. For a single bit, the code is simply 0 followed by 1 . To create a code of $d+1$ bits, given the code of $d$ bits: first place the $2^{d}$ bit strings of the dimension $d$ in order. Concatenate a 0 onto the left end of each of the bit strings. Then repeat the same $2^{d}$ bit strings in reverse order, concatenating a 1 onto the left end of each one. Figure 2.1 shows the BRGC for up to three bits. Note that the last bit string in the code differs from the first in a single bit, making the code a cyclic Gray code. 


\begin{tabular}{llllll}
0 & 0 & 0 & 0 & 0 & 0 \\
1 & 0 & 1 & 0 & 0 & 1 \\
& 1 & 1 & 0 & 1 & 1 \\
& 1 & 0 & 0 & 1 & 0 \\
\hline 1 & 1 & 0 \\
1 & 1 & 1 \\
& & & 0 & 1 \\
& & & 0 & 0
\end{tabular}

Figure 2.1: The standard Binary Reflected Gray Code for 1, 2, and 3 dimensions

\subsection{Quasi-Gray codes}

A further generalization of Gray codes was provided by Fredman [Fre78] when he coined the term quasi-Gray code. In Fredman's work, a quasi-Gray code was defined to be a $d$-dimensional bit string along with some unbounded additional data structure. The quasi-Gray code differs in each successive state in one bit of the $d$-dimensional bit string, but the algorithm may also make arbitrary changes to its additional data structure. In order to efficiently generate the successor bit string in a quasi-Gray code, the algorithm may make use of the additional data structure, reading fewer bits than it would otherwise need to. Fredman characterizes the efficiency of a generating algorithm by the worst-case number of bits read and written to generate each bit string in the code. Under this model, the algorithm will write a single bit in the worst case if and only if the algorithm does not use any additional data structure. In this case, Fredman notes that it would be generating a Gray code.

We modify this definition slightly to improve the clarity of analysis. Because the additional data structure and the code itself are equally part of the algorithm's state, we consider Fredman's additional data structure to be a part of the generated code. We use the term quasi-Gray code to refer to a sequence of $d$-dimensional bit strings where successive bit strings each differ by at most a constant number of bits. This is similar to Fredman's model, but with a stronger limitation on the number of bits 
being written, as his model allowed an arbitrary number of bits to be written while still considering it a quasi-Gray code. Thus an algorithm that, under Fredman's definition, generated a $d$-dimensional quasi-Gray code using an additional $k$ bits of data structure and writing at most a constant number of bits would, in our redefinition of the term, simply generate a $(d+k)$-dimensional quasi-Gray code.

Fredman's results generate codes while writing in the worst case $O\left(2^{d}\right)$ bits, which do not qualify as quasi-Gray codes under our definition of the term, and which have space efficiency $O\left(1 / 2^{2 d}\right)$.

Rahman and Munro [RM08] begin to address space efficiency while generating quasi-Gray codes, without naming it explicitly, which we continue in this thesis. However, we expand upon their work by also examining the average number of bits read by generating algorithms. Rahman and Munro construct quasi-Gray codes in the manner of Fredman, where they generate a $d$-dimensional Gray code, while keeping some additional data structure. However, the authors use much smaller data structures, eventually constructing an algorithm that requires only three extra bits. This brings the space efficiency of their algorithm up to $1 / 8$, which was the first algorithm to read less than $d$ bits in the worst case with space efficiency that did not become arbitrarily close to 0 for large $d$. This algorithm generates a $d$-dimensional quasi-Gray code while reading $\log (d-3)+6$ bits in the worst case and writing at most 7 bits.

Rahman and Munro do not use the DAT model, and they sometimes include in their analysis the amount of work to decode rankings inside the quasi-Gray code's structure. Under the DAT model, the rank can be embedded into the tree, so we don't need to examine such costs. For this reason we compare our work to theirs only in the case where they also ignore these costs. Rahman and Munro give one such algorithm, which ignores the work involved in determining ranks. The bounds given for this algorithm also hold in the DAT model. The algorithm uses only a single bit of extra data structure, giving it a space efficiency of $1 / 2$. This algorithm generates a $d$-dimensional quasi-Gray code while reading $\log (d-1)+4$ bits in the worst case and writing at most 4 bits.

Rahman and Munro also consider the problem of adding or subtracting two numbers, when each is stored using a quasi-Gray code representation. They give a data 
structure that uses $d=n+O\left(\log ^{2} n\right)$ bits. Incrementing or decrementing a number on its own, equivalent to generating the next or previous bit string in the quasi-Gray code, requires at most $O(\log n)$ bits to be read and at most 5 bits to be changed. This data structure also supports adding or subtracting two such numbers of dimension $d$ and $d^{\prime}$, where $d \geq d^{\prime}$, while reading at most $O\left(d+\log d^{\prime}\right)$ bits.

Savage highlights works around Gray codes in her survey [Sav97], which includes both Gray codes as sequences of bit strings, and more general combinatorial sequences with minimal change between successive states, referred to as combinatorial Gray codes. The majority of the work related to quasi-Gray codes for bit strings discusses mathematical aspects of Gray codes such as existence of various classes of Gray codes, rather than algorithms for efficient generation of these sequences.

Frank Ruskey devotes a chapter of his book-in-progress [Rus01] to algorithms for generating combinatorial Gray codes. The majority of this work is devoted to generating other forms of combinatorial Gray codes than bit strings as we consider in this thesis, but he does include a section on generating the BRGC. In this section, Ruskey describes an algorithm to generate the $d$-dimensional BRGC while reading and writing $O(1)$ bits in the worst case, by making use of an additional $d$ bits. This gives a quasi-Gray code with space efficiency of $1 / 2^{d}$.

Knuth, in volume 4 of The Art of Computer Programming [Knu05], discusses the problem of generating Gray codes. He gives an overview of various applications for Gray codes, and surveys some known results, both in generating them, and in analysis of other properties. Knuth shows an algorithm for loopless generation of a Gray code of dimension $d$, [BER76] where each generating step can be executed without any loop in the algorithm, that has space efficiency $2^{-d}$. He also discusses other properties of Gray codes, such as a balanced number of bit flips for each bit position, having each bit keep its value for at least a constant number of states, or monotonicity, where each bit string of rank $x$ in the code has at most as many bits set to 1 as the bit string of rank $x+2$.

Frandsen et al. [SFMS97] describe a method of generating a sequence of bit strings of dimension $d=n+\log n$ while reading and writing at most $O(\log n)$ bits for each generating step. Their counter algorithm has space efficiency $O(1 / n)$, which 
converges to 0 in the limit $n \rightarrow \infty$. An observation by Brodal [Bro09] improves the space efficiency of their counter algorithm to $1 / 2$, matching the efficiency of the work by Rahman and Munro. We improve on these counters in Section 4.5 in terms of average bits read and space efficiency.

\subsection{Upper and lower bounds}

We use the Decision Assignment Tree (DAT) model to analyze algorithms for generating bit strings in a quasi-Gray code. The model was first introduced for this context by Fredman [Fre78]. We will describe the DAT model and how we use it in detail in Chapter 3, while briefly describing it here.

An algorithm to generate a quasi-Gray code takes a bit string of dimension $d$ as input, and modifies it to become the next bit string in the quasi Gray-code. This operation does not necessarily require reading all $d$ bits of the current string. The DAT model can be used for proving both upper and lower bounds on the required number of bits to be read in order to generate a quasi-Gray code. In this work, we construct and analyze our algorithms under this model to provide upper bounds.

Meanwhile, a non-trivial lower bound for the worst case number of bits read while generating a Gray code remains unknown. A trivial DAT, such as iterating through the standard binary representations of 0 to $2^{d}-1$, in the worst case, will require reading and writing all $d$ bits to generate the next bit string, but it may also read and write as few as one bit when the least-significant bit changes. On average, it reads and writes $2-2^{1-d}$ bits. Meanwhile, it is possible to create a DAT that generates the Binary Reflected Gray Code, as described in Section 3.3. This DAT would always write exactly one bit, but requires reading all $d$ bits to generate each successive bit string in the code. This is because the least-significant bit is flipped if and only if the parity is even, which can only be determined by reading all $d$ bits.

To generate a Gray code of dimension $d$ with length $L=2^{d}$, Fredman [Fre78] uses the DAT model to show that any algorithm will require reading $\Omega(\log d)$ bits for some bit string. Fredman conjectures that for a Gray code of dimension $d$ with $L=2^{d}$, any DAT will have to read all $d$ bits to generate at least one bit string in the code. 
That is, any DAT generating the code must have height $d$. This remains an open problem. $^{1}$

\footnotetext{
${ }^{1}$ In [RM08] the authors claim to have proven this conjecture true for "small" $d$ by exhaustive search.
} 


\section{Chapter 3}

\section{Decision Assignment Trees}

\subsection{The Decision Assignment Tree Model}

In the DAT model, an algorithm is described as a binary tree. We say that a DAT which reads and generates bit strings of length $d$ has dimension $d$. Further, we refer to the bit string that the DAT reads and modifies as the state of the DAT. Generally the initial bit string for a quasi-Gray code of dimension $d$, and thus the initial state of its generating DAT, is the bit string made up of a sequence of $d$ zeros. Each internal node of the tree is labeled with a single fixed position $0 \leq i \leq d-1$ within the input bit string, and represents reading that bit $i$. Figure 3.1 shows a DAT that generates the BRGC of dimension $d=3$. The BRGC that is generated is also seen in Figure 2.1 .

Let $T$ be a DAT of dimension $d$. The algorithm starts at the root of $T$, and reads the bit with which that node is labeled. Then it moves to a left or right child of that node, depending on whether the bit read was a 0 or a 1 , respectively. This repeats recursively until a leaf node in the tree is reached.

Each leaf node of $T$ represents a subset of states where the bits read along the path to the leaf are in a fixed state. More formally, a state $S_{i}$ for a DAT is represented by a single leaf $L_{i}$. When the DAT is in state $S_{i}$, traversing the DAT while reading the current state will lead to the leaf $L_{i}$. It is possible for two different states $S_{i}$ and $S_{j}$ to share the same leaf $L_{m}$ if they both cause the same bit positions to be read and 


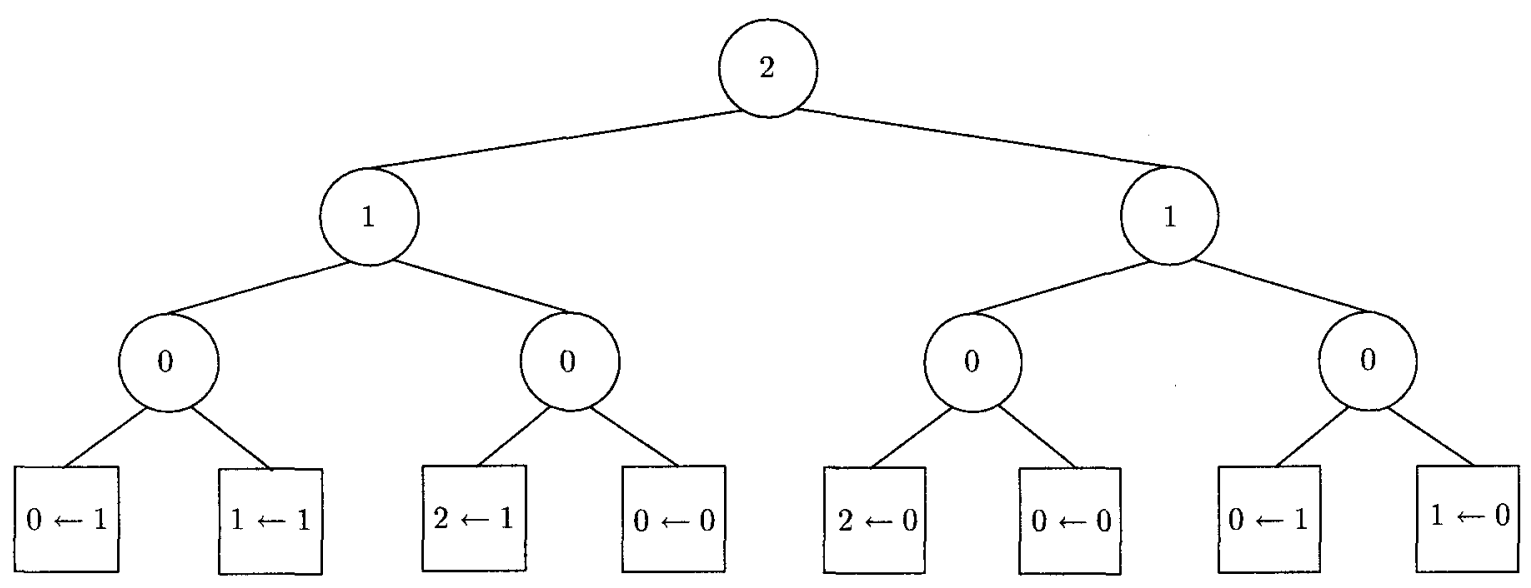

Figure 3.1: A DAT that generates the Binary Reflected Gray Code on three bits. The bits are labelled from 0 as the right-most to 2 as the left-most bit. The label in each internal node specifies which bit is being read. Control moves to the left child or right child if the bit is equal to 0 or 1 , respectively. The rules in the leaf nodes set a bit $i$ to a new value, 0 or 1 .

those positions share all the same values. In this case it is required at least one of the bits that were not read on the path to the leaf $L_{m}$ must be in a different state each time the DAT traversal reaches $L_{m}$.

The leaf nodes each contain rules that describe which bits to update to generate the next bit string in the code. The update rules are constrained in the following ways:

1. Each rule must set a single fixed bit directly to 0 or to 1 .

2. The rules together must change at least one bit.

Analysis under this model can be done by examining the structure of the tree. The worst-case number of bits read will be equal to the height of the tree, and the worst-case number of bits written will be equal to the maximum number of rules in any leaf of the tree. The average number of bits read and written are not easily derived from the tree's structure. The average number of bits read will be equal to the sum of all paths in the tree, weighted by the fraction of times the path is used 
when generating the entire quasi-Gray code. The average number of bits written will be a similar weighted average, for the number of rules in the leaves.

\subsection{Assembling DATs}

Decision Assignment Trees can be assembled by joining together other DATs. We present here observations based on this.

Lemma 3.1. Let $L$ and $R$ each be a DAT for a binary code of dimension d with space efficiency 1 . The $L$ and $R$ trees may be joined together, under a new root node, to create a DAT of dimension $d+1$ with space efficiency 1 .

Proof. We join the $L$ tree and $R$ tree together by adding a new root node, and making $L$ and $R$ its left and right subtrees respectively. The subtrees $L$ and $R$ each read and write $d$ bits. We assign them to the same bits, 0 to $d-1$, and the root node to the $d$-th bit. We assume w.l.o.g. that reading a 0 at the root node means to move to the root of the $L$ subtree, while 1 means to move to the root of the $R$ subtree. Assume that the $d$-th bit is initially set to 0 . If it is 1 , then swap $L$ and $R$ in what follows.

It is clear that $L$ remains a valid Decision Assignment Tree of dimension $d$. And because it never changes the $d$-th bit, it will never cause the $R$ subtree to be used. Thus our initial construction is a valid Decision Assignment Tree of dimension $d+1$ that counts through only $2^{d}$ states.

Let $L_{A}$ be the first state of $L$ and $L_{Z}$ be the last state. If $L$ is cyclic, then any two states such that $L_{A}$ immediately follows $L_{Z}$ in the code are valid. Similarly, let $R_{A}$ and $R_{Z}$ be the first and last states of $R$. We modify the construction to join the code generated by $L$ to the code generated by $R$, producing a new code of dimension $d+1$ :

1. Make the update rules of $L_{Z}$ change the counter to state $R_{A}$, and change the d-th bit to 1 .

2. Make the update rules of $R_{Z}$ change the counter to state $L_{A}$, and change the d-th bit to 0 . 
Note that the leaf for state $L_{Z}$ may be shared by another state $L_{x}$, and is therefore invoked multiple times by the $L$ subtree. If this is the case, simply split the leaf node, giving it two children that differentiate on a bit that is different in $L_{Z}$ and $L_{x}$. Repeat this process until the leaf for $L_{Z}$ is not used for any other states in $L$.

Because the $L$ subtree is able to count through $2^{d}$ states, it will take $2^{d}-1$ steps to go from $L_{A}$ to $L_{Z}$, and likewise for the $R$ subtree. Within each of these steps, the $d$-th bit is not changed and the subtree is able to operate correctly. After generating $2^{d}-1$ bit strings in $L$, the $d$-th bit is changed, and the state is changed to $R_{A}$. This makes $2^{d}$ consecutive bit strings, generated by the $L$ subtree. Now the same argument holds for the $R$ subtree, which will generate $2^{d}-1$ bit strings within its own subtree, and then move to $L_{A}$, completing a full cycle through all $2^{d+1}$ possible bit strings. Thus, we have a cyclic binary code of dimension $d+1$ and space efficiency 1 .

\subsection{Generating the BRGC}

Lemma 3.2. The Binary Reflected Gray Code of dimension d can be generated by a DAT, which requires reading $d$ bits and writing at most 1 bits to generate each successive bit string.

Proof. The proof is by induction. For a Binary Reflected Gray Code of dimension 1 , create a DAT with height 1 . At the root node, the single bit is read. If the root reads a 0 , move to its left child, if it reads a 1 , move to its right child. The left child changes bit 0 to 1 and the right child changes bit 0 to be 0 . This generates the cyclic BRGC of dimension 1.

Let $L$ be a DAT for the BRGC of dimension $d$, and let $R$ be a DAT which generates the same bit strings as $L$ in reverse order. Then, by Lemma 3.1, we can use $L$ and $R$ to construct a new DAT of dimension $d+1$. We choose $L_{A}$ to be the bit string $000 \ldots 0$ and $L_{Z}$ to be the bit string $1000 \ldots 0$. Since the BRGC is cyclic, $L_{A}$ is the state which follows $L_{Z}$. We choose $R_{A}$ to be the state $1000 \ldots 0$ and $R_{Z}$ to be $000 \ldots 0$.

Because $R_{Z}=L_{A}$ and $L_{Z}=R_{A}$, no bits need to be changed to move between them. Thus, in the combined DAT, only the $(d+1)$-th bit needs to change in order to generate $L_{A}$ from $R_{Z}$ or $R_{A}$ from $L_{Z}$, and the DAT is able to move between subtrees 
with only one bit changed. Further, the first $2^{d}$ states will correspond to a dimension $d$ BRGC, with a 0 in the $(d+1)$-th bit. The second $2^{d}$ states will correspond to a dimension $d$ BRGC in reverse order, with a 1 in the $(d+1)$-th bit. This is precisely the definition of the BRGC of dimension $d+1$. Thus, we are able to construct a DAT that generates the BRGC of any dimension.

An example of a DAT that generates the BRGC for dimension $d=3$ can be seen in Figure 3.1. 


\section{Chapter 4}

\section{Efficient generation of quasi-Gray codes}

In this chapter we address how to efficiently generate quasi-Gray codes of dimension d. We examine efficiency in terms of the number of bits read and written in the worst case to generate each successive bit string, the number of bits read on average to generate each successive bit string while generating the entire code, and the space efficiency. The codes we generate are all cyclic. First we present DATs that read up to $d$ bits in the worst case, but read fewer bits on average. Then we present our lazy counters that read at most $O(\log d)$ bits in the worst case, while also reading fewer bits on average.

\subsection{Recursive Partition Gray Code (RPGC)}

We show a method for generating a cyclic Gray code of dimension $d$ that requires reading an average of $6 \log d$ bits to generate each successive bit string. First, assume that $d$ is a power of two for simplicity. In this special case, we show that it reads on average no more than $4 \log d$ bits to generate each bit string in the code. We use both an increment and decrement operation to generate the Gray code, where the increment operation generates the next bit string of dimension $d$ in the code, and the decrement operation generates the previous bit string of dimension $d$ in the code. 
Both increment and decrement operations are defined recursively, and they make use of each other. Pseudocode for these operations is provided in Algorithms 1 and 2. To generate the next bit string in the code, we partition the bit string of dimension $n$ into two substrings, $A$ and $B$, each of dimension $n / 2$. We then recursively increment $A$ unless $A=B$, that is, unless the bits in $A$ are in the same state as the bits in $B$, at which point we recursively decrement $B$. Testing $A=B$ is done by reading and comparing sequential pairs of bits in $A$ and $B$ until a pair is found that differ. In the analysis, we will see that this test reads only a constant number of bits on average.

To generate the previous bit string in the code, we again partition the bit string of dimension $n$ into two substrings, $A$ and $B$, each of dimension $n / 2$. We then recursively decrement $A$ unless $A=B+1$, that is, the bits of $A$ are in the same state as the bits of $B$ would be after an increment operation, at which time we recursively increment $B$ instead. Testing $A=B+1$ can be done by simulating an increment of $B$ and testing $A$ for equality against the result. In the analysis, we will see that this test also reads only a constant number of bits on average.

Figure 4.1 shows a conceptualization of the Recursive Partition Gray Code. Wheels $A$ and $B$ represent the states in part $A$ and $B$ of the code, respectively. When $A$ is incremented, it moves to the next clockwise location, and when $B$ is decremented it moves to the next counter-clockwise location. And inside each wheel $A$ and $B$ is another set of wheels.

The increment and decrement operations must partition the bit strings identically; we assume w.l.o.g. that the $A$ partition contains the first $n / 2$ bits, and $B$ contains the remaining $n / 2$ bits. Pseudocode for the increment (RecurIncrementPow2) and decrement (RecurDecrementPow2) operations follows. 


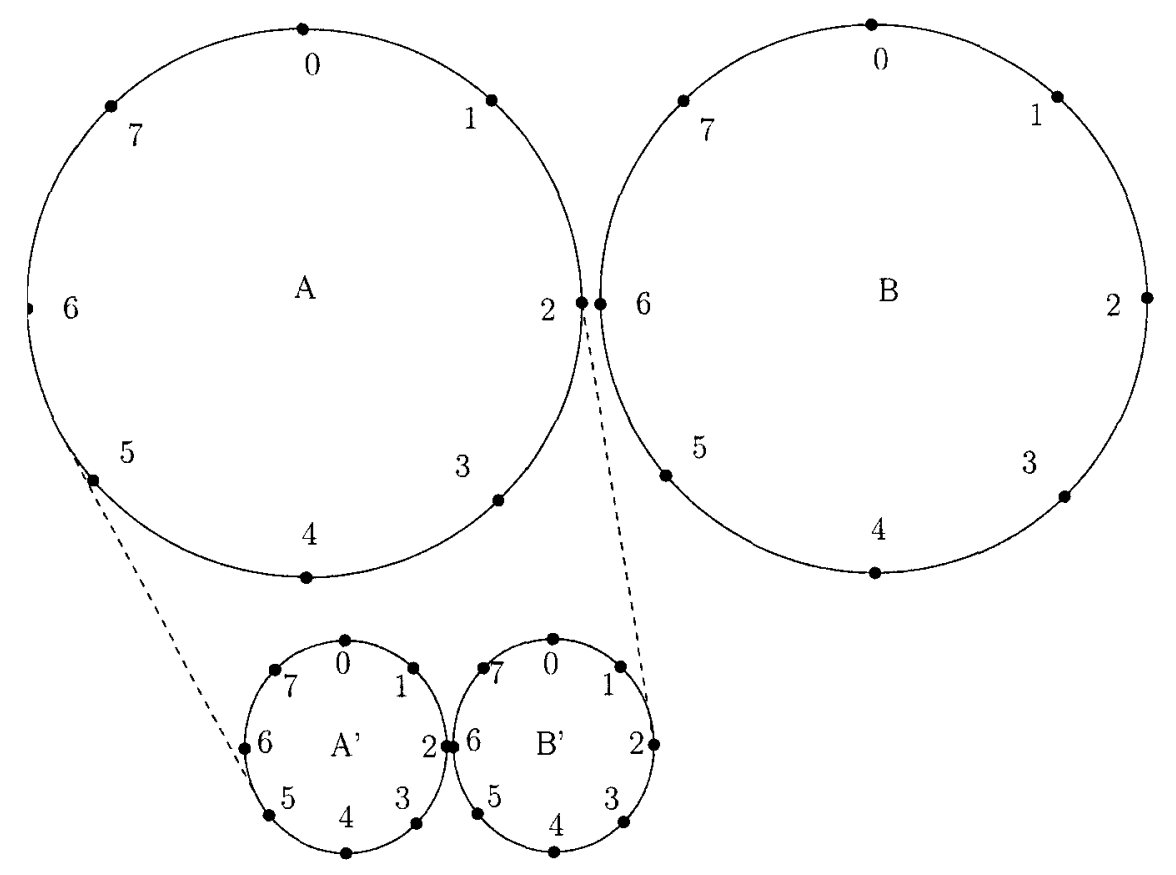

Figure 4.1: Conceptualization of the Recursive Partition Gray Code. A moves clockwise while $A \neq B$, at which point $B$ moves counter-clockwise.

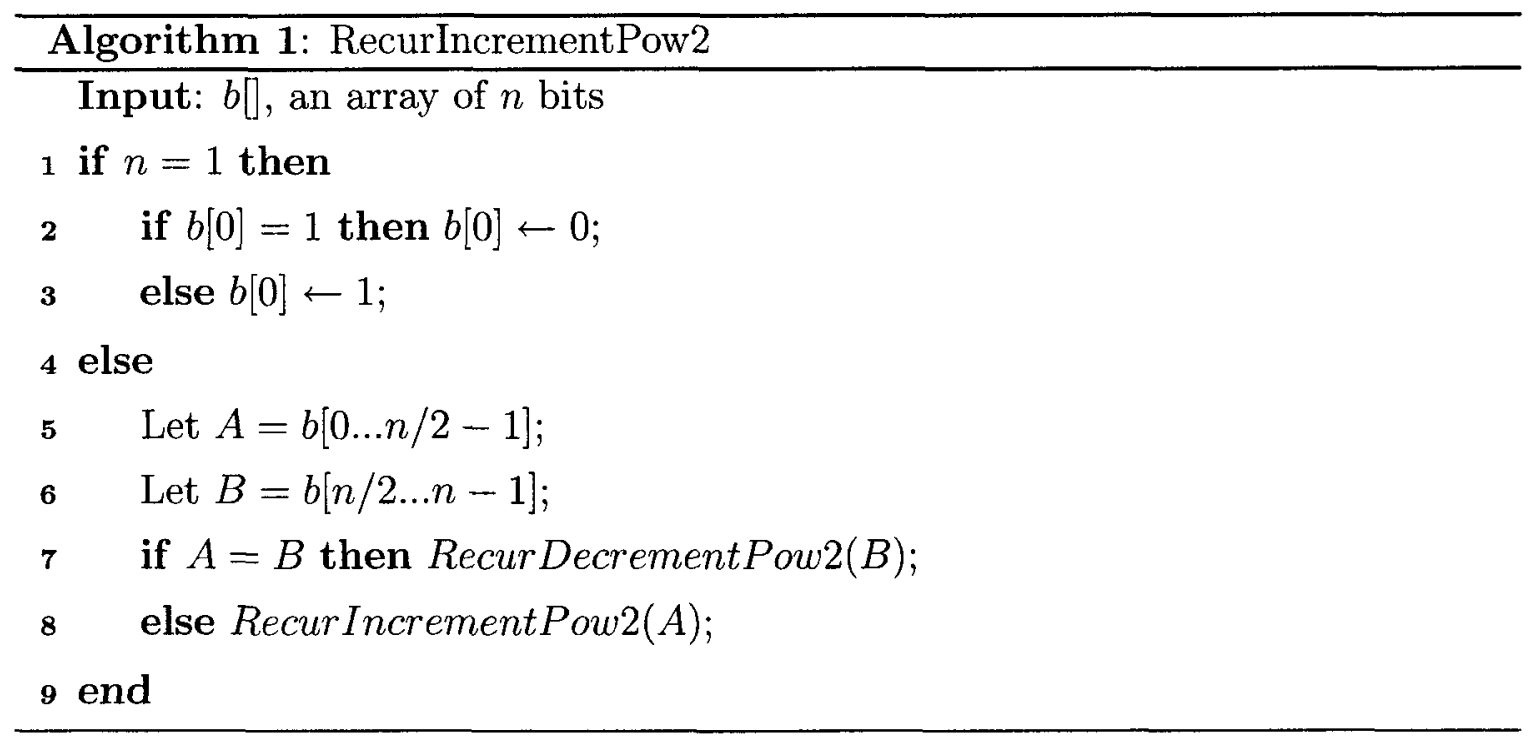




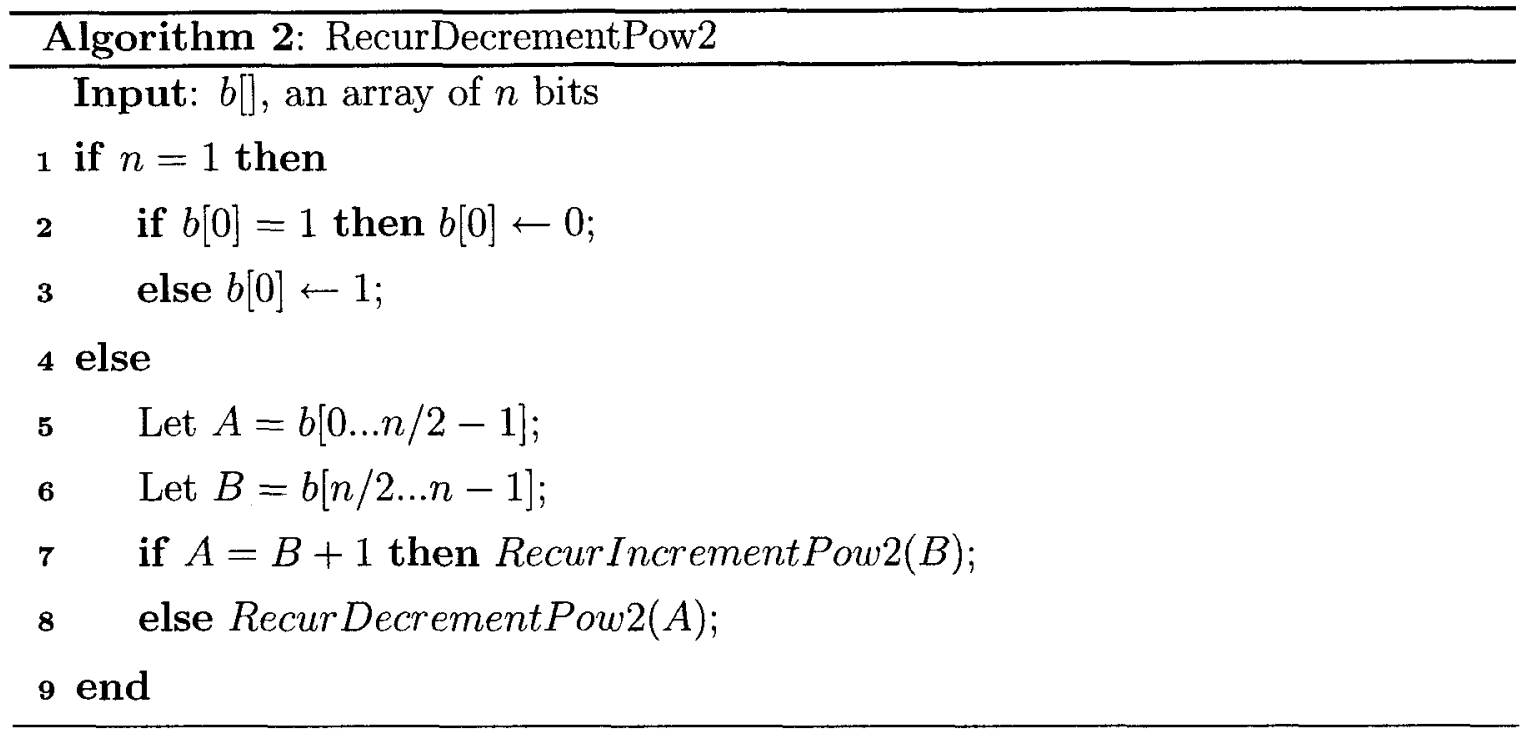

Lemma 4.1. The Recursive Partition Gray Code algorithm can be performed by a DAT to generate a Gray code of dimension d when d is a power of two.

Proof. We will recursively define a DAT $I_{d}$ that performs the RecurIncrementPow2 operation, and a DAT $D_{d}$ that performs the RecurDecrementPow2 operation.

Let $C_{d}$ be a DAT that compares two bit strings of dimension $d$ to determine if they differ in at least one bit. Each leaf of $C_{d}$ represents a single bit $i$ of the bit strings, such that $i$ is the first bit seen which differs. Assume the DAT scans the bit strings for differences from bit 0 to bit $d-1$ in order. Then number the leaves such that leaf $C_{d, i}$ is reached when bit $i$ is the first bit seen that differs between the two bit strings. Leaf $C_{d, d}$ is reached when there is no difference between the two bit strings.

Let $M_{d}$ be a DAT that compares two bit strings of dimension $d$, to determine if they differ in at least two bits. As the root of $M_{d}$, use a $C_{d}$ DAT. At leaves $C_{d, i}$, $0 \leq i \leq d-2$, place another DAT $C_{d-i-1}$, that compares the last $d-i-1$ bits between the two bit strings. Call the leaves of these trees $C_{d, i, j}$ when they are the $j$-th leaf of the subtree rooted at the $i$-th leaf of the top-level $C_{d}$ DAT. Then some leaf $C_{d, i, j}$, for $0 \leq i \leq d-2$ and $j \leq d-i-1$, will be reached when the two bit strings differ in at least two bits, while some leaf $C_{d, i, j}$, for $i \geq d-1$ or $j \geq d-i$, will be reached when the two bit strings have less than two bits which differ. Call the first set of leaves the 2-differ leaves, and the latter the 0 -differ leaves. 
$I_{1}$ and $D_{1}$ have a height of 1 , and are each constructed identically. The root node reads the single bit and control transfers to a child. The leaf nodes flip the value of the single bit: if the bit was 1 it writes a 0 , and if the bit was 0 it writes a 1 .

To construct a DAT for $I_{d}$, with $d>1$, place a DAT $C_{d / 2}$ as the root. At each leaf except $C_{d / 2, d / 2}$, put the root node of an $I_{d / 2}$ DAT. At the leaf $C_{d / 2, d / 2}$, place the root node of a $D_{d / 2}$ DAT.

To construct a DAT for $D_{d}$, with $d>1$, place a DAT $M_{d / 2}$ as the root, which considers the entire bit string of dimension $d$. At each 0-differ leaf in the $M_{d / 2}$ DAT, place the root of a $I_{d / 2}$ DAT, which simulates an increment operation on the last $d / 2$ bits, with each leaf being the root of a $C_{d / 2}$ DAT. At each leaf of the $C_{d / 2}$ DATs, place the root of a $D_{d / 2}$ DAT that operates also on the last $d / 2$ bits, with exception that the highest rank leaf in each $C_{d / 2}$ DAT is the root of a $I_{d / 2}$ DAT that operates on the first $d / 2$ bits instead. Finally, at each 2-differ leaf node, place a $D_{d / 2}$ DAT that operates on the last $d / 2$ bits.

At the end of this process, look at each path from the root to a leaf in the final DAT. Let $i$ and $j$ be nodes on any such path such that $i$ and $j$ both read the same bit of the input string. Assume without loss of generality that $j$ is in the left subtree of $i$. Then remove $j$ and its right subtree from the DAT, replacing $j$ with its left child. Continue this process until there are no such $i$ and $j$ in the DAT.

Lemma 4.2. For a dimension $d \geq 1$, the RecurIncrementPow2 algorithm generates a Gray code of dimension d with length $2^{d}$.

Proof. The proof is by induction on $d$.

Let $d=1$, then the RecurIncrementPow2 algorithm flips the bit twice, creating a Gray code of length $2=2^{1}=2^{d}$. The same is true for the RecurDecrementPow2 algorithm.

Then assume it is true for dimensions less than $d$. We will show that for dimension $d$, the RecurIncrementPow2 algorithm generates a Gray code of length $2^{d}$.

Let $|X|$ be the dimension of a bit string $X$.

If $d$ is even, and $A=B$ initially, then the algorithm starts by decrementing $B$. For each decrement of $B$, the algorithm will increment $A 2^{d / 2}-1$ times in order to 
make them equal again. This comes from the fact that $|A|=d / 2<d$ and thus the bits move through $2^{d / 2}$ unique states.

$B$ is decremented $2^{d / 2}$ times before it reaches its initial state again, since $|B|=$ $d / 2<d$. Since only one of $A$ and $B$ is changed at each step, the total number of bit strings generated is equal to the number of times $B$ is decremented plus the number of times $A$ is incremented. This is $2^{|B|}+2^{|B|} \cdot\left(2^{|A|}-1\right)=2^{d / 2}+2^{d / 2}\left(2^{d / 2}-1\right)=$ $2^{d / 2}+2^{d}-2^{d / 2}=2^{d}$. The same holds for RecurDecrementPow2 by a symmetric argument.

At each step if the algorithm, bits are read but not written, except in the base case, where $d=1$. Each generating step recurses on one sub problem, thus only one bit is written, and the resulting code is a Gray code.

Therefore, the RecurIncrementPow2 algorithm generates a Gray code of dimension $d$ with length $L=2^{d}$.

Theorem 4.3. Let $d \geq 2$ be a power of two. There exists a DAT that generates a Gray code of dimension $d$ and length $L=2^{d}$, where generating the next bit string requires reading on average no more than $4 \log d$ bits of the current string. In the worst case, $d$ bits are read, and only 1 bit is written.

Proof. By Lemma 4.1, we can construct a DAT that performs the RecurIncrementPow2 and RecurDecrementPow2 operations and generates a Recursive Partition Gray code of dimension $d$.

Since the RPGC has length $L=2^{d}$, the algorithm will be executed once for each possible bit string of dimension $d$. Based on this observation, we bound the average number of bits read by studying the expected number of bits read given a random bit string of dimension $d$. The proof is by induction on $d$. For the base case $d=2$, in the worst case we read at most 2 bits, so the average number of bits read in a random bit string is at most $2 \leq 4 \log d$. Then we assume our claim is true for a random bit string of dimension $d / 2$.

We define $|X|$ to denote the dimension of a bit string $X$. Let $C(A, B)$ be the number of bits read to determine whether or not $A=B$, where $A$ and $B$ are bit strings and $|A|=|B|$. Let $I(X)$ be the number of bits read to increment the bit 
string $X$. Let $D(X)$ be the number of bits read to decrement the bit string $X$. Note that since we are working in the DAT model, we read any bit at most one time, and $D(X) \leq|X|$.

To finish the proof, we need to show that $\mathrm{E}[I(X)] \leq 4 \log d$, when $X$ is a random bit string of dimension $d$.

We can determine the expected value of $C(A, B)$ as follows. $C(A, B)$ must read two bits at a time, one from each of $A$ and $B$, and compare them, only until it finds a pair that differs. Given two random bit strings, the probability that bit $i$ is the first bit that differs between the two strings is $1 / 2^{i+1}$. If the two strings differ in bit $i$, then the function will read exactly $i+1$ bits in each string. If $|A|=|B|=n / 2$, then the expected value of $C(A, B)$ is

$$
\mathrm{E}[C(A, B)]=2 \sum_{i=0}^{n / 2-1} \frac{i+1}{2^{i+1}}=2 \sum_{i=1}^{n / 2} \frac{i}{2^{i}}=2\left(\frac{2^{n / 2+1}-n / 2-2}{2^{n / 2}}\right)=2\left(2-\frac{n / 2+2}{2^{n / 2}}\right)
$$

Let $X=A B$, and $|A|=|B|=d / 2$. Then $|X|=d$.

For a predicate $P$, we define $\mathbf{1}_{P}$ to be the indicator random variable whose value is 1 when $P$ is true, and 0 otherwise.

Note that $I(A)$ is independent of $\mathbf{1}_{A=B}$ and $\mathbf{1}_{A \neq B}$. This is because the relation between $A$ and $B$ has no effect on the distribution of $A$ (which remains uniformly distributed among all bit strings of dimension $d / 2)$. The same is true of $I(B), D(A)$ and $D(B)$.

The RecurIncrementPow2 operation only performs one increment or decrement action, depending on the condition $A=B$, thus the expected number of bits read by $I(X)$ is

$$
\begin{aligned}
\mathrm{E}[I(X)] & =\mathrm{E}[C(A, B)]+\mathrm{E}\left[\mathbf{1}_{A=B} D(B)\right]+\mathrm{E}\left[\mathbf{1}_{A \neq B} I(A)\right] \\
& \leq 2\left(2-\frac{d / 2+2}{2^{d / 2}}\right)+\frac{1}{2^{d / 2}} \frac{d}{2}+\left(1-\frac{1}{2^{d / 2}}\right) \mathrm{E}[I(A)] \\
& \leq 4-\frac{d / 2+4}{2^{d / 2}}+4 \log (d / 2) \\
& \leq 4+4 \log d-4 \log 2 \\
& =4 \log d
\end{aligned}
$$


as required.

Last, in the worst case when $A=B$, the comparison test will require reading all bits in $A$ and $B$, and thus all $d$ bits in the code.

Now consider the case when $d$ is not a power of two. When the increment operation is given a bit string with an even number of bits, the algorithm is the same as in the power of two case. However, when it is given a bit string with an odd number of bits, it uses the first bit as a direction bit. While the direction bit is 0 , the increment operation will recursively increment the remaining bits, until they reach their state of highest rank. At that point, the increment operation flips the direction bit to 1 . From then on, while the direction bit is 1 , the increment operation will recursively decrement the remaining bits, until they reach their state of minimum rank. Then the increment operation would flip the direction bit back to 0 , reaching its initial state. This generates all possible states for the bit string.

The decrement operation functions similarly to the increment operation when given a bit string with an odd number of bits, except that it decrements the remaining bits when the direction bit is 0 and increments them when the direction bit is 1 .

Pseudocode for the increment (RecurIncrement) and decrement (RecurDecrement) operations for the more general scenario follows. 


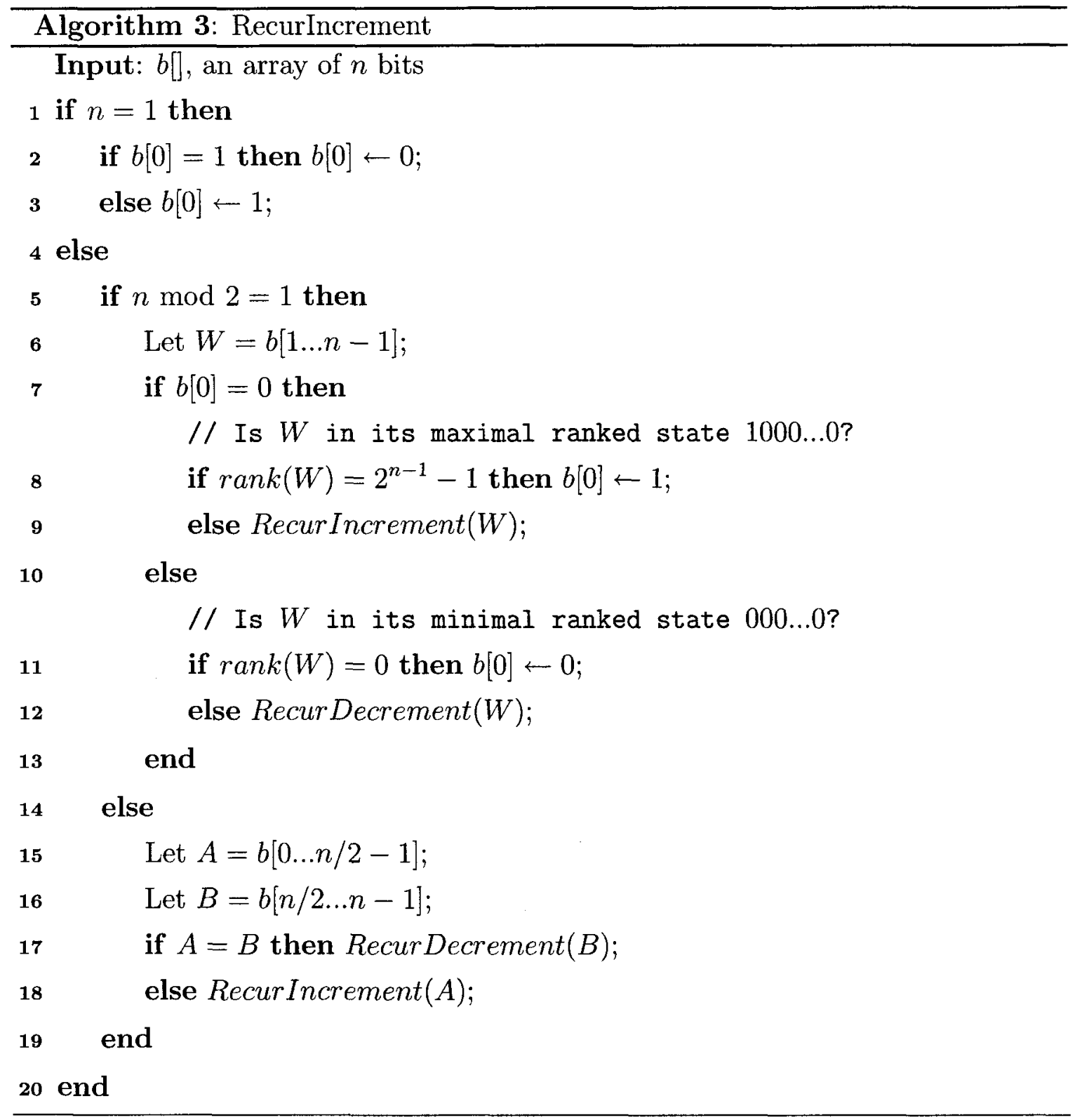




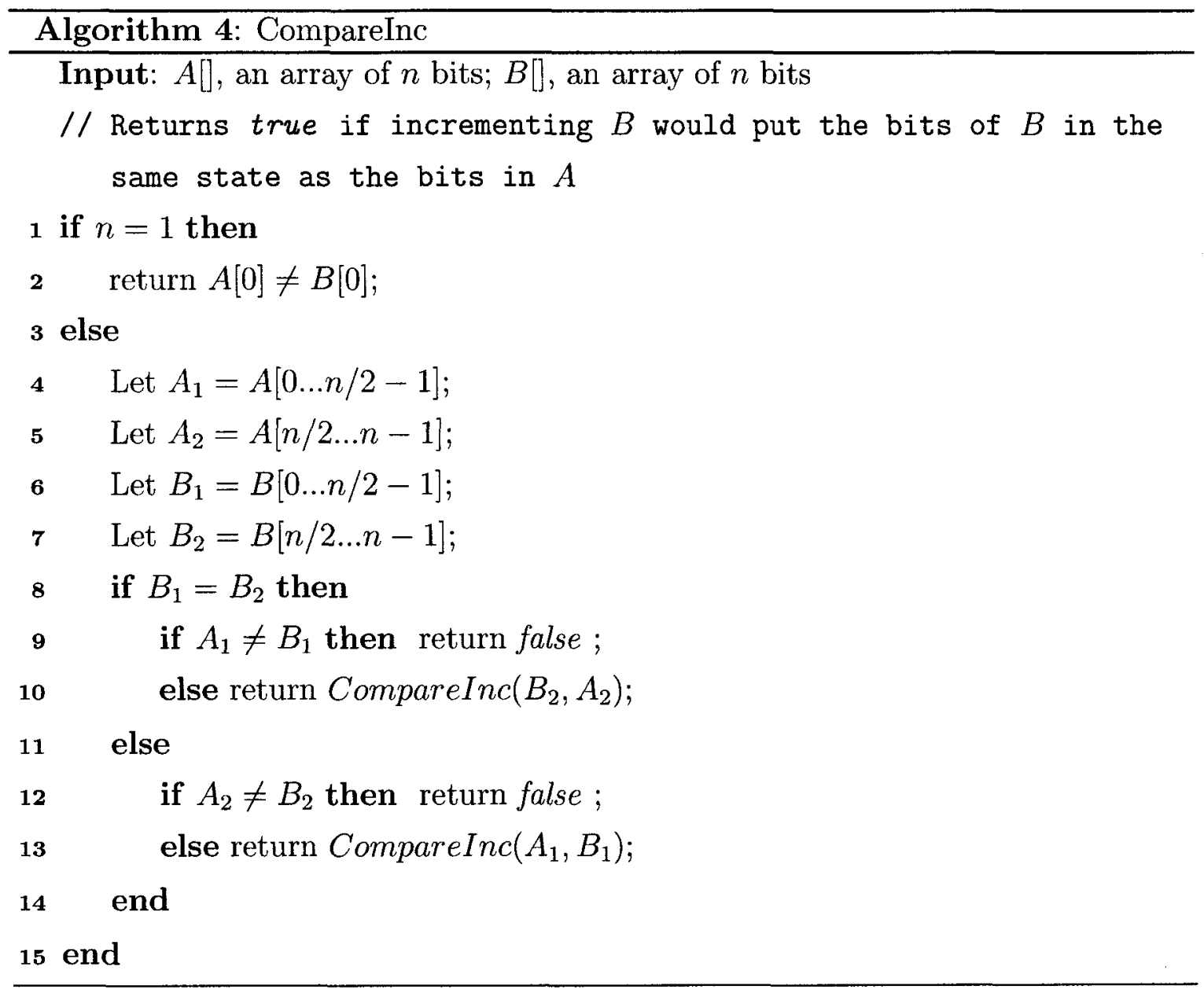




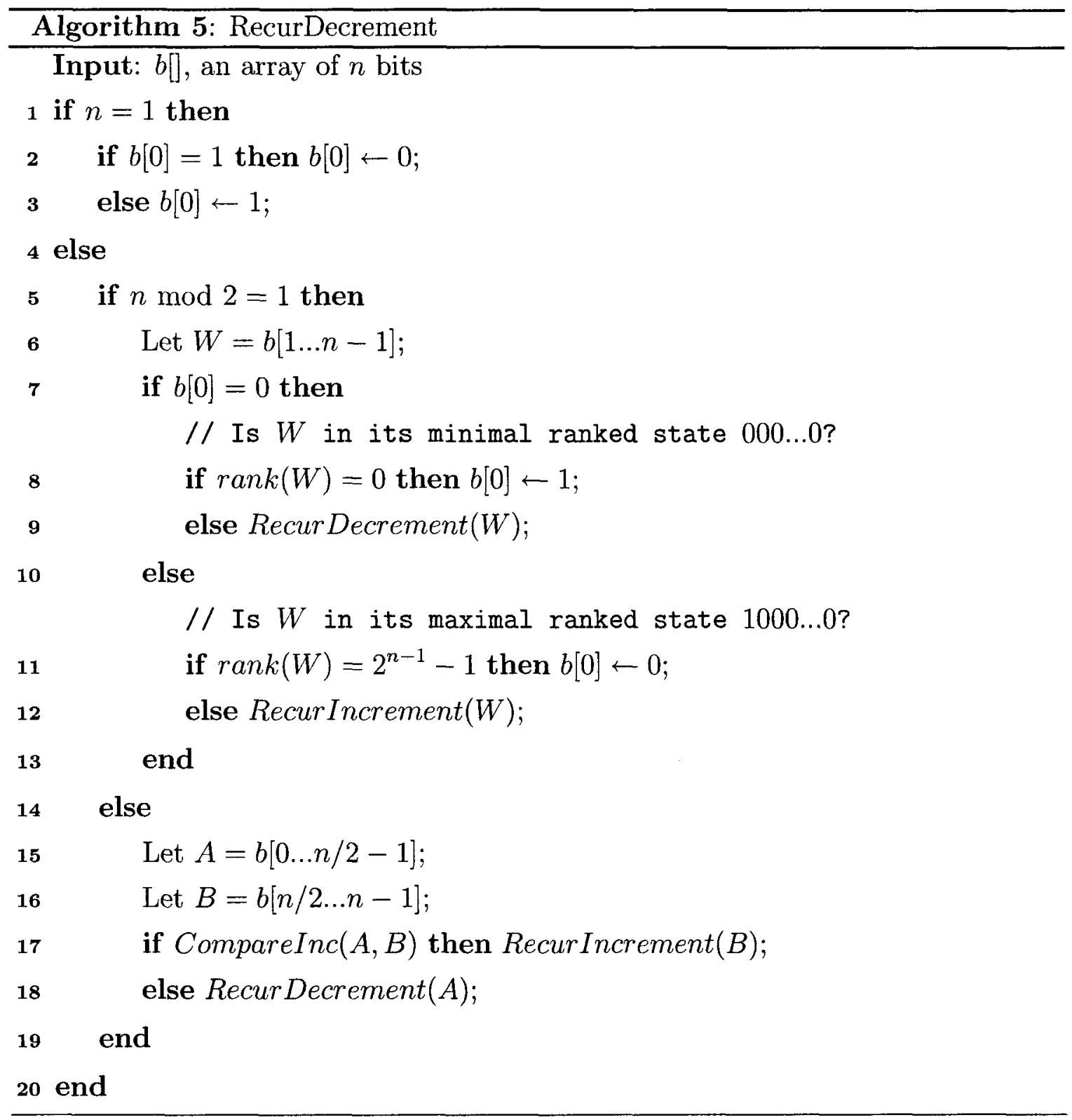

Lemma 4.4. For a dimension $d \geq 1$, the Recursive Partition Gray Code algorithm generates a Gray code of dimension d with length $2^{d}$.

Proof. The proof is by induction on $d$.

Let $d=1$, then the RecurIncrement algorithm flips the bit twice, creating a Gray code of length $2=2^{1}=2^{d}$. The same is true for the RecurDecrement algorithm. 
Then assume it is true for dimensions less than $d$. We will show that for dimension $d$, the RecurIncrement algorithm generates a Gray code of length $2^{d}$.

If $d$ is even, and $A=B$ initially, then the algorithm starts by decrementing $B$. For each decrement of $B$, the algorithm will increment $A 2^{d / 2}-1$ times in order to make them equal again. This comes from the fact that $|A|=d / 2<d$ and thus the bits move through $2^{d / 2}$ unique states.

$B$ is decremented $2^{d / 2}$ times before it reaches its initial state again, since $|B|=$ $d / 2<d$. Since only one of $A$ and $B$ is changed at each step, the total number of bit strings generated is equal to the number of times $B$ is decremented plus the number of times $A$ is incremented. This is $2^{|B|}+2^{|B|} \cdot\left(2^{|A|}-1\right)=2^{d / 2}+2^{d / 2}\left(2^{d / 2}-1\right)=$ $2^{d / 2}+2^{d}-2^{d / 2}=2^{d}$. The same holds for RecurDecrement by a symmetric argument.

If $d$ is odd, then the algorithm will increment the last $d-1$ bits until they reach their maximally ranked state. Since $d-1<d$, this will generate $2^{d-1}-1$ bit strings. Next, the algorithm will flip the first bit, one bit string, and decrement the last $d-1$ bits until they reach their initial state. This generates another $2^{d-1}-1$ bit strings. Finally, it flips the first bit back to its initial state as well. In total, this generates $2^{d-1}-1+1+2^{d-1}-1+1=2^{d}$ bit strings. The RecurDecrement algorithm performs the same operations in a different order, producing the same number of bit strings.

At each step if the algorithm, bits are read but not written, except in the base case, where $d=1$. Each generating step recurses on one sub problem, thus only one bit is written, and the resulting code is a Gray code.

Therefore, the Recursive Partition Gray Code algorithm generates a Gray code of dimension $d$ with length $L=2^{d}$.

Theorem 4.5. Let $d \geq 2$. There exists a DAT that generates a Gray code of dimension $d$ and length $L=2^{d}$, where generating the next bit string requires reading on average no more than $6 \log d$ bits of the current string. Only 1 bit is written in the worst case, and d bits are read.

Proof. From Lemma 4.4, we know the length of a RPGC of dimension $d$ is exactly $2^{d}$. Then, as in the proof of Theorem 4.3, the algorithm will be executed once for each possible bit string of dimension $d$. As such, we bound the average number of 
bits read by studying the expected number of bits read given a random bit string of dimension $d$. The proof is by induction on $d$. We will show that the expected number of bits read to increment is at most $5.623 \log d$. In some cases, incrementing requires decrementing a substring of the code, and we will show that in these cases, the expected number of bits read by a decrementing step is at most $7.746 \log d$ bits.

For the base case $d=2$, in the worst case we read at most 2 bits to perform RecurIncrement, so the average bits read is at most $2 \leq 5.623 \log d$. Similarly to perform RecurDecrement, we read at most 2 bits, and thus read on average at most $2 \leq 7.746 \log d$.

Then we assume that these both hold for a random bit string of dimension less than $d$. Let $I(X)$ and $D(X)$ be the number of bits read to increment or decrement the bit string $X$, respectively. To finish the proof, we will show that $\mathrm{E}[I(X)] \leq 5.623 \log d$, when $X$ is a random bit string of dimension $d$.

We define $|X|$ to denote the dimension of the bit string $X$, and $X^{-t}$ refers to a substring of $X$ with dimension $|X|-t$. Let $C(A, B)$ be the number of bits read to determine whether or not $A=B$, where $A$ and $B$ are bit strings and $|A|=$ $|B|=n / 2$. Let $M(A, B)$ be the number of bits read by the CompareInc algorithm, which determines whether or not $A=B+1$, where $A$ and $B$ are bit strings and $|A|=|B|=n / 2$. Note that since we are working in the DAT model, we read any bit at most one time. And finally, let $I=3.5$ and $D=7.746$.

The expected value of $C(A, B)$ is given in the proof of Theorem 4.3 as $\mathrm{E}[C(A, B)]=$ $2\left(2-\frac{2+n / 2}{2^{n / 2}}\right)$, and we can determine the expected value of $M(A, B)$ as follows. $M(A, B)$ compares two bit strings against a third, all of equal dimension, $n / 4$. There are four possible outcomes for the CompareInc function. We examine the cost and probability of each to determine the expected value of $M(A, B)$.

When $B_{1}=B_{2} \neq A_{1}$, CompareInc returns false. In this case $B_{1}$ is compared against $B_{2}$ and $A_{1}$. Since $B_{1}=B_{2}$, we read all $n / 2$ bits in $B_{1}$ and $B_{2}$. Since $A_{1}$ differs from the others, we expect to read $\sum_{i=1}^{n / 4} \frac{i}{2^{i}}=2-\frac{2+n / 4}{2^{n / 4}}$ bits of $A_{1}$, by the same argument as made for $C(A, B)$. Thus we read at most $2+n / 2$ bits on average in this case, which happens with probability $\frac{\left(2^{n / 4}\right)\left(2^{n / 4}\right)\left(2^{n / 4}-1\right)}{2^{n}}=\frac{2^{3 n / 4}-2^{n / 2}}{2^{n}}=$ 
$\frac{2^{n / 4}-1}{2^{n / 2}}$

When $B_{1} \neq B_{2}$ and $B_{2} \neq A_{2}$, CompareInc returns false again. In this case $B_{2}$ is compared against $B_{1}$ and against $A_{2}$. If the two comparisons read the bits of $B_{2}$ in the same order, the second comparison will read many of the same bits as the first. In the DAT model, this means we can count far fewer bits read on average for the second comparison. The expected number of bits read in each of $B_{1}$ and $A_{2}$ is $2-\frac{2+n / 4}{2^{n / 4}}$. The number of bits read in $B_{2}$ is the max of the number of bits read in $B_{1}$ and $A_{2}$. Consider an equivalent problem. Given two random bit strings of dimension $k$, if we examine bit positions in both strings together, count the number of positions we expect to look at to find at least one 1 in each string. The probability that position $i, 1 \leq i \leq k$, is the first position where we have seen a 1 at some position $j \leq i$ in each string is $2 \frac{2^{2(k-i)}\left(2^{i}-1\right)}{2^{2 k}}-\frac{2^{2(k-i)}}{2^{2 k}}=2 \frac{2^{i}-1}{2^{2 i}}-\frac{1}{2^{2 i}}$. The first term counts the number of strings with the first $i-1$ bits set to 0 and the $i$-th bit set to 1 for one string, and at least one 1 somewhere in the first $i$ bits of the other. It is multiplied by two, since this can occur with a 1 in position $i$ for either of the two strings. The second term keeps us from double counting the case where the first 1 in both strings occurs at position $i$. The expected number of bit positions read is then

$$
\sum_{i=1}^{k} i\left(2 \frac{2^{i}-1}{2^{2 i}}-\frac{1}{2^{2 i}}\right)=2 \sum_{i=1}^{k}\left(\frac{i}{2^{i}}-\frac{3}{2} \frac{i}{2^{2 i}}\right) \leq 8 / 3
$$

which is the expected number of bits read in $B_{2}$. The above summation converges to $8 / 3$ in the limit $i \rightarrow \infty$, and so we can upper bound it by this value. Thus, in this case, the average number of bits we read is at most $20 / 3$. This scenario occurs with probability $\frac{\left(2^{n / 4}\right)^{2}\left(2^{n / 4}-1\right)^{2}}{2^{n}}=\frac{2^{n / 2}-2^{1+n / 4}+1}{2^{n / 2}}=1-\frac{2^{1+n / 4}-1}{2^{n / 2}}$.

In the other two cases, CompareInc recurses on a problem of size $n / 2$. Since there are $n$ bits in the input to the $M(A, B)$, we can bound the average number of bits read in this case by $n$, which happens with probability $\frac{\left(2^{n / 4}\right)\left(2^{n / 4}\right)}{2^{n}}+\frac{\left(2^{n / 4}\right)^{2}\left(2^{n / 4}-1\right)}{2^{n}}=$ $\frac{1}{2^{n / 4}}$.

By combining these four cases, the expected value of $M(A, B)$ is

$$
\mathrm{E}[M(A, B)]=\left(\frac{2^{n / 4}-1}{2^{n / 2}}\right)(2+n / 2)+\left(1-\frac{2^{1+n / 4}-1}{2^{n / 2}}\right)(20 / 3)+\left(\frac{1}{2^{n / 4}}\right) n \leq 7.507,
$$


for $n \geq 2$. This bound comes from maximizing the function, where according to Maple, the function has a maximum value $\approx 7.506883004$, for $n \geq 2$.

For a predicate $P$, we define $\mathbf{1}_{P}$ to be the indicator random variable whose value is 1 when $P$ is true, and 0 otherwise.

Let $A$ and $B$ be two random bit strings of equal dimension. Note that $I(A)$, $I(B), D(A)$, and $D(B)$ are all independent of $\mathbf{1}_{A=B}$ and $\mathbf{1}_{A=B+1}$. This is because the relation between $A$ and $B$ has no effect on the distribution of $A$ or $B$ (which each remain uniformly distributed among all bit strings of their dimension).

Throughout the following cases, we assume that the first bit of $A$ or $B$, if we recurse on $A$ or $B$ respectively, will always be read in the next recursive step, so we are able to read the bit for free in the current step. This is true, since we count the first bit explicitly in each inductive step, and the base case reads all of its bits, including the first.

Let $X$ be an input string of dimension $d$. We consider the cases when $d$ is an even or odd number separately. First, assume that $d$ is an even number.

The RecurIncrement operation only performs one increment or decrement action, depending on the condition $A=B$. We bound the cost of $D(B)$ here by $d / 2$ which is an upper bound for the DAT model. The comparison in $C(A, B)$ will read the first bit of $A$ and $B$, so at least one bit will be double counted, which we can subtract. Thus the expected number of bits read by $I(X)$, when $|X|$ is even, is

$$
\begin{aligned}
\mathrm{E}[I(X)] & =\mathrm{E}[C(A, B)]-1+\mathrm{E}\left[\mathbf{1}_{A=B} D(B)\right]+\mathrm{E}\left[\mathbf{1}_{A \neq B} I(A)\right] \\
& \leq 2\left(2-\frac{d / 2+2}{2^{d / 2}}\right)-1+\frac{1}{2^{d / 2}} \frac{d}{2}+\left(1-\frac{1}{2^{d / 2}}\right) \mathrm{E}[I(A)] \\
& =3-\frac{d / 2+4}{2^{d / 2}}+\mathrm{E}[I(A)] \\
& \leq 3+I \log (d / 2) \\
& \leq I \log d,
\end{aligned}
$$

because $I=3.5 \geq 3$.

We also examine the cost of the RecurDecrement operation, as we will need it for the case when $d$ is odd. The RecurDecrement operation performs one decrement or increment action, depending on the condition $A=B+1$. We make sure that we 
count both the first bit of $A$ and $B$ so that we can subtract one bit as being double counted. The first bit of $B$ is always read by $M(A, B)$, but the first bit of $A$ is not read when $B_{1} \neq B_{2}$. We bound the cost of $I(B)$ here by $d / 2$, giving us the expected number of bits read by $D(X)$, when $|X|$ is even, as

$$
\begin{aligned}
\mathrm{E}[D(X)] & =\mathrm{E}[M(A, B)]+\mathrm{E}\left[\mathbf{1}_{B_{1} \neq B_{2}} 1\right]-1+\mathrm{E}\left[\mathbf{1}_{A=B+1} I(B)\right]+\mathrm{E}\left[\mathbf{1}_{A \neq B+1} D(A)\right] \\
& \leq 7.507+\frac{1}{2^{d / 4}}-1+\frac{d / 2}{2^{d / 2}}+\mathrm{E}[D(A)] \\
& \leq 6.507+\frac{2^{d / 4}+d / 2}{2^{d / 2}}+\mathrm{E}[D(A)] \\
& \leq 7.715+D \log (d / 2) \\
& \leq D \log d
\end{aligned}
$$

because $D=7.746 \geq 7.715$. Elementary algebraic manipulation shows that $\frac{2^{d / 4}+d / 2}{2^{d / 2}}$ is at most $\frac{\sqrt{2}+1}{2} \leq 1.208$.

Now consider the case when $d$ is an odd number. Let $X=x A B$, where $|x|=1$ and $|A|=|B|=(d-1) / 2$. We assume that the bit $x$ can be read for free, which we will later show is the case.

When $x=0$, the RecurIncrement operation checks to see that $A B \neq 100 \ldots 0$, and increments $A B$ when it is true, otherwise it stops and reads no more bits. Let $a_{i}$ and $b_{j}$ be the $i$-th and $j$-th bits of $A$ and $B$, respectively. We assume that the comparison of $A B$ to the bit string $100 \ldots 0$ is done by looking at the bits of $A$ and of $B$ in an alternating sequence, such that the first bit read is $a_{0}$, then $b_{0}$, followed by $a_{1}$ and $b_{1}$, and so on.

Consider the four cases for the possible values of $a_{0}$ and $b_{0}$, the first bits read.

Case $a_{0}=0, b_{0}=0$ : Then we know $A B \neq 100 \ldots 0$ and $A B$ will be incremented. The first step of incrementing $A B$ is to test $A=B$. This test will read the first bit of $A$ and $B$, and so at least one bit is double-counted and can be subtracted here. Thus, in this case, the expected number of bits read will be at most $2-1+\mathrm{E}\left[C\left(A^{-1}, B^{-1}\right)\right]+$ $\mathrm{E}\left[\mathbf{1}_{A^{-1} \neq B^{-1}} I(A)\right]+\mathrm{E}\left[\mathbf{1}_{A^{-1}=B^{-1}} D(B)\right]=1+4-2 \frac{2+(d / 2-1)}{2^{d / 2-1}}+\mathrm{E}\left[\mathbf{1}_{A^{-1} \neq B^{-1}} I(A)\right]+$ $\mathrm{E}\left[\mathbf{1}_{A^{-1}=B^{-1}} D(B)\right]=5-\frac{3+d / 2}{2^{d / 2-1}}+\mathrm{E}\left[\mathbf{1}_{A^{-1} \neq B^{-1}} I(A)\right]+\mathrm{E}\left[\mathbf{1}_{A^{-1}=B^{-1}} D(B)\right]$. 
Case $a_{0}=1, b_{0}=1$ : This case has the same analysis and expected value as the previous case.

Case $a_{0}=1, b_{0}=0$ : Then we must continue checking if $A B=100 \ldots 0$. This will read an expected $2-(2+(d-2)) 2^{-(d-2)}$ bits. We already know the result of the test $A=B$ from the bits $a_{0}$ and $b_{0}$, so no more bits need to be read in order to proceed. And we can subtract one bit as we double count the first bit of $A$ or $B$ once again. Thus, in this case, the expected number of bits read will be at most $2-1+2-\frac{2+(d-2)}{2^{d-2}}+\mathrm{E}\left[\mathbf{1}_{A^{-1} \neq B^{-1}} I(A)\right]+\mathrm{E}\left[\mathbf{1}_{A^{-1}=B^{-1}} D(B)\right]=3-\frac{d}{2^{d-2}}+$ $\mathrm{E}\left[\mathbf{1}_{A^{-1} \neq B^{-1}} I(A)\right]+\mathrm{E}\left[\mathbf{1}_{A^{-1}=B^{-1}} D(B)\right]$.

Case $a_{0}=0, b_{0}=1$ : Then we know $A B \neq 100 \ldots 0$ and also that $A \neq B$, and we do not read any more bits to continue to the next recursive step. We have also double counted the first bit of $A$ or $B$ and can subtract it. Thus, in this case, the expected number of bits read will be at most $2-1+\mathrm{E}\left[\mathbf{1}_{A^{-1} \neq B^{-1}} I(A)\right]+\mathrm{E}\left[\mathbf{1}_{A^{-1}=B^{-1}} D(B)\right]=$ $1+\mathrm{E}\left[\mathbf{1}_{A^{-1} \neq B^{-1}} I(A)\right]+\mathrm{E}\left[\mathbf{1}_{A^{-1}=B^{-1}} D(B)\right]$.

We bound the number of bits read for $D(B)$ by $d / 2$, as this is an upper bound for the DAT model. Because each of these cases occurs once out of every four executions, the total expected value of $I(X)$ when $|X|$ is odd and $x=0$ is

$$
\begin{aligned}
\mathrm{E}\left[I_{x=0}(X)\right] \leq & \frac{1}{2}\left(5-\frac{3+d / 2}{2^{d / 2-1}}\right)+\frac{1}{4}\left(3-\frac{d}{2^{d-2}}\right)+\frac{1}{4}(1) \\
& +\mathrm{E}\left[\mathbf{1}_{A \neq B} I(A)\right]+\mathrm{E}\left[\mathbf{1}_{A=B} D(B)\right] \\
\leq & \frac{14}{4}-\frac{3+d / 2}{2^{d / 2}}-\frac{d}{2^{d}}+\frac{d / 2}{2^{d / 2}}+\mathrm{E}[I(A)] \\
= & \frac{14}{4}-\frac{3}{2^{d / 2}}-\frac{d}{2^{d}}+\mathrm{E}[I(A)] \\
\leq & \frac{14}{4}+I \log (d / 2) \\
= & I \log d,
\end{aligned}
$$

because $I=3.5=14 / 4$.

Next we consider when $x=1$. In this scenario, the RecurIncrement operation checks to see that $A B \neq 000 \ldots 0$, and decrements $A B$ when it is true, otherwise it stops and reads no more bits. Let $a_{i}$ and $b_{j}$ be the $i$-th and $j$-th bits of $A$ and $B$, respectively. In this case, we do the comparison of $A B$ to the bit string $000 \ldots 0$ in a 
different order. We first look at the bit $b_{0}$, then $b_{n / 2}$, followed by $b_{1}$ and $b_{n / 2+1}$. We continue reading $B$ in this alternating order until all bits have been read. Then we check the bits $a_{n / 2}, a_{n / 2+1}$, up to $a_{n-1}$, and finally $a_{0}, a_{1}$, up to $a_{n / 2-1}$.

Consider the four cases for the possible values of $b_{0}$ and $b_{n / 2}$, the first bits read. And note that $M(A, B)$ always reads $b_{0}$ and $b_{n / 2}$ so we do not count them outside of that function.

Case $b_{0}=1, b_{n / 2}=0$ : Then we know that $A B \neq 000 \ldots 0$. The decrement operation requires checking that $A \neq B+1$, using the CompareInc function. This number of bits read by this function is $M(A, B)$. We also count the first bit of $A$ if $B_{1} \neq B_{2}$ and it is not read by $M(A, B)$, so that it is double-counted and we can subtract it. Thus, in this case, the expected number of bits read will be at most $\mathrm{E}[M(A, B)]+\mathrm{E}\left[\mathbf{1}_{B_{1} \neq B_{2}} 1\right]-1+\mathrm{E}\left[\mathbf{1}_{A \neq B+1} D(A)\right]+\mathrm{E}\left[\mathbf{1}_{A=B+1} I(B)\right] \leq 7.507+\frac{1}{2^{d / 4}}-1+$ $\mathrm{E}\left[\mathbf{1}_{A \neq B+1} D(A)\right]+\mathrm{E}\left[\mathbf{1}_{A=B+1} I(B)\right] \leq 7.215+\mathrm{E}\left[\mathbf{1}_{A \neq B+1} D(A)\right]+\mathrm{E}\left[\mathbf{1}_{A=B+1} I(B)\right]$. This follows from the fact that $\frac{1}{2^{d / 4}}$ is a decreasing function for $d \geq 2$, and its maximum value, at $d=2$, is $2^{-1 / 2} \leq 0.708$.

Case $b_{0}=0, b_{n / 2}=1$ : This case has the same analysis and expected value as the previous case.

Case $b_{0}=1, b_{n / 2}=1$ : This case also has the same analysis and expected value as the previous two cases.

Case $b_{0}=0, b_{n / 2}=0$ : Then we need to continue checking if $A B \neq 000 \ldots 0$, and if it is true, $A B$ will be decremented. We note that this check reads bits in the same order as $M(A, B)$ compares the bit strings $A$ and $B$. While the check continues to find all 0 bits, the function CompareInc would also find equality, so we know that it would read at least as many bits as we check to find a bit set to 1 . This holds until $3 n / 4$ bits have been read, which is the most that $M(A, B)$ will read. When these bits are all 0 , we must continue reading the last $n / 4$ bits to compare them against $000 \ldots 0$. This happens for $2^{n / 4}$ of the possible $2^{n}$ inputs, thus with probability $2^{-3 n / 4}$. To compare the last $n / 4$ bits against $000 \ldots 0$, we expect to read $2-\frac{2+n / 4}{2^{n / 4}}$ bits. We also make sure the first bit of $A$ is double-counted, as when $B_{1} \neq B_{2}$, it will not be read by $M(A, B)$ or by the comparison to $000 \ldots 0$. Thus, in this case, the expected number of bits read 
will be at most $\mathrm{E}[M(A, B)]+\mathrm{E}\left[\mathbf{1}_{B_{1} \neq B_{2}} 1\right]-1+\frac{1}{2^{3 n / 4}}\left(2-\frac{2+d / 4}{2^{d / 4}}\right)+\mathrm{E}\left[\mathbf{1}_{A \neq B+1} D(A)\right]+$ $\mathrm{E}\left[\mathbf{1}_{A=B+1} I(B)\right] \leq 7.507+\frac{1}{2^{d / 4}}-1+\frac{2}{2^{3 d / 4}}-\frac{2+d / 4}{2^{d}}+\mathrm{E}\left[\mathbf{1}_{A \neq B+1} D(A)\right]+\mathrm{E}\left[\mathbf{1}_{A=B+1} I(B)\right]$.

We bound the number of bits read for $I(B)$ by $d / 2$, as this is an upper bound for the DAT model. If $|A|$ is even, we can bound the expected cost of $D(A)$ as shown above. When $|A|$ is odd, then $D(A)$ will have the same expected number of bits read as $I(A)$, since they both increment half the time, and decrement half the time. Thus, in both cases, we are able to bound $\mathrm{E}[D(A)]$ by $7.746 \log (d / 2)$ by induction. Because each of these cases occurs once out of every four executions, the total expected value of $I(X)$ when $|X|$ is odd and $x=1$ is

$$
\begin{aligned}
\mathrm{E}\left[I_{x=1}(X)\right] \leq & \frac{3}{4}(7.215)+\frac{1}{4}\left(7.507-1+\frac{1}{2^{d / 4}}+\frac{2}{2^{3 d / 4}}-\frac{2+d / 4}{2^{d}}\right) \\
& +\mathrm{E}\left[\mathbf{1}_{A \neq B+1} D(A)\right]+\mathrm{E}\left[\mathbf{1}_{A=B+1} I(B)\right] \\
\leq & 7.038+\frac{1}{4}\left(\frac{1}{2^{d / 4}}+\frac{2}{2^{3 d / 4}}-\frac{2+d / 4}{2^{d}}\right)+\frac{d / 2}{2^{d / 2}}+\mathrm{E}\left[\mathbf{1}_{A \neq B+1} D(A)\right] \\
= & 7.038+\frac{1}{4}\left(\frac{1}{2^{d / 4}}+\frac{2}{2^{3 d / 4}}-\frac{2+d / 4}{2^{d}}+\frac{2 d}{2^{d / 2}}\right)+\mathrm{E}\left[\mathbf{1}_{A \neq B+1} D(A)\right] \\
\leq & 7.038+\frac{2.832}{4}+\mathrm{E}[D(A)] \\
\leq & 7.746+D \log (d / 2) \\
= & D \log d,
\end{aligned}
$$

because $D=7.746$. This follows from maximizing the function $\frac{1}{2^{d / 4}}+\frac{2}{2^{3 d / 4}}-\frac{2+d / 4}{2^{d}}+$ $\frac{4 \cdot d / 2}{2^{d / 2}}$, which according to Maple, has a maximum value $\approx 2.831931894$ for $d \geq 2$.

Last, we must consider the bit $x$. While generating the entire Gray code, the bit $x$ will be 0 for half of the time, and 1 the other half. Thus $I_{x=0}(X)$ will execute half of the time when $|X|$ is odd, and $I_{x=1}(X)$ will execute the other half. Therefore, the 
expected value of $I(X)$ when $|X|$ is odd is

$$
\begin{aligned}
\mathrm{E}[I(X)] & \leq \mathrm{E}\left[\mathbf{1}_{x=0} I_{x=0}(X)\right]+\mathrm{E}\left[\mathbf{1}_{x=1} I_{x=1}(X)\right] \\
& \leq \frac{1}{2}(I \log d)+\frac{1}{2}(D \log d) \\
& =\frac{1}{2}(3.5 \log d)+\frac{1}{2}(7.746 \log d) \\
& \leq 5.623 \log d
\end{aligned}
$$

because $I=3.5$ and $D=7.746$. From our initial choice of $I$ and $D$, all of the above holds, and we have shown that in all cases, for a bit string $X$ of dimension $d$, $\mathrm{E}[I(X)] \leq 5.623 \log d$

This satisfies the inductive proof, however, we did not count the cost of reading the bit $x$ when the input string is odd. Note that when we recurse on a substring $A$ or $B$ of $X$, we have compared $A=B$ (to increment) or $A=B+1$ (to decrement), each of which requires reading at least one bit of $A$ and $B$. Since this bit will be $x$ when $A$ or $B$ is odd, we will have always already read the bit $x$ during the previous recursive step. Therefore, the bit $x$ can be read for free whenever we are inside a recursive call. Only at the first level of recursion must we count reading the bit $x$. For any $d$, the algorithm's recursion eventually reaches the base case of $d=2$. In this case, the worst case number of bits read will be 2 , but we count it as $6 \log d=6$. This leaves $6-2=4$ bits counted in our average that we did not actually read. These bits more than account for the one bit $x$ at the top level of the recursion.

Therefore, to generate the next bit string in the Recursive Partition Gray Code, given an input string $X$, the RecurIncrement algorithm will read on average $\mathrm{E}[I(X)] \leq$ $6 \log d$ bits.

\subsection{Composite code construction}

In this section, we show a method to take a code with space efficiency 1 that reads on average $r$ bits, and construct a new, larger, code that reads on average $O(\log \log r)$ bits, while maintaining the same space efficiency, but increasing the worst-case number of bits written by one. 
Lemma 4.6. Let $d \geq 1, r \geq 3, p \geq 1, w \geq 1$ be integers. Assume we have a DAT for a code of dimension d, that generates $L=2^{d}$ bit strings, such that the following holds: Given a bit string of length d, generating the next bit string in the code requires reading no more than $r$ bits on average, reads $p$ bits in the worst case, and writes at most $w$ bits in the worst case.

Then there is a space-optimal DAT for a code of dimension $d+\lceil\log r\rceil$, where generating each bit string from the previous one requires reading no more than $6 \log \lceil\log r\rceil+$ 3 bits on average, reading $p+\lceil\log r\rceil$ bits in the worst case, and writing at most $w+1$ bits. That is, the average number of bits read decreases from $r$ to $O(\log \log r)$, while the worst-case number of bits written increases by one.

Proof. We are given a DAT $A$ that generates a code of dimension $d$. The DAT, $A$, requires reading on average no more than $r$ bits, and writing at most $w$ bits to generate each bit string. We construct a DAT $B$ for the Recursive Partition Gray Code of dimension $d^{\prime}$, such as described in Section 4.1. The DAT, $B$, requires reading $\leq 6 \log d^{\prime}$ bits on average to generate the next state, and requires writing only 1 bit in the worst case.

We construct a new DAT from the two DATs of dimension $d$ and $d^{\prime}$. The combined DAT generates bit strings of dimension $d+d^{\prime}$. The last $d^{\prime}$ bits of the combined code, when updated, will cycle through the code generated by $B$. The first $d$ bits, when updated, will cycle through the code generated by $A$.

The DAT initially moves the last $d^{\prime}$ bits through $2^{d^{\prime}}$ states according to the rules of $B$. When it leaves this final state, to generate the initial bit string of $B$ again, the DAT also moves the first $d$ bits to their next state according to the rules of $A$.

Figure 4.2 shows a conceptualization of the Composite code. Wheels $A$ and $B$ represent the states in part $A$ and $B$ of the code, respectively. $B$ moves clockwise around its wheel with each increment operation. When $B$ moves from its highest state back to 0 , then $A$ also moves one step in the clockwise direction.

During each generating step, the last $d^{\prime}$ bits are considered and moved to their next state in the code generated by the rules of $B$, and checked to see if they have reached their initial position, The first $d$ bits are only incremented when the last $d^{\prime}$ bits cycle back to their initial state - once for every $2^{d^{\prime}}$ bit strings generated by the 

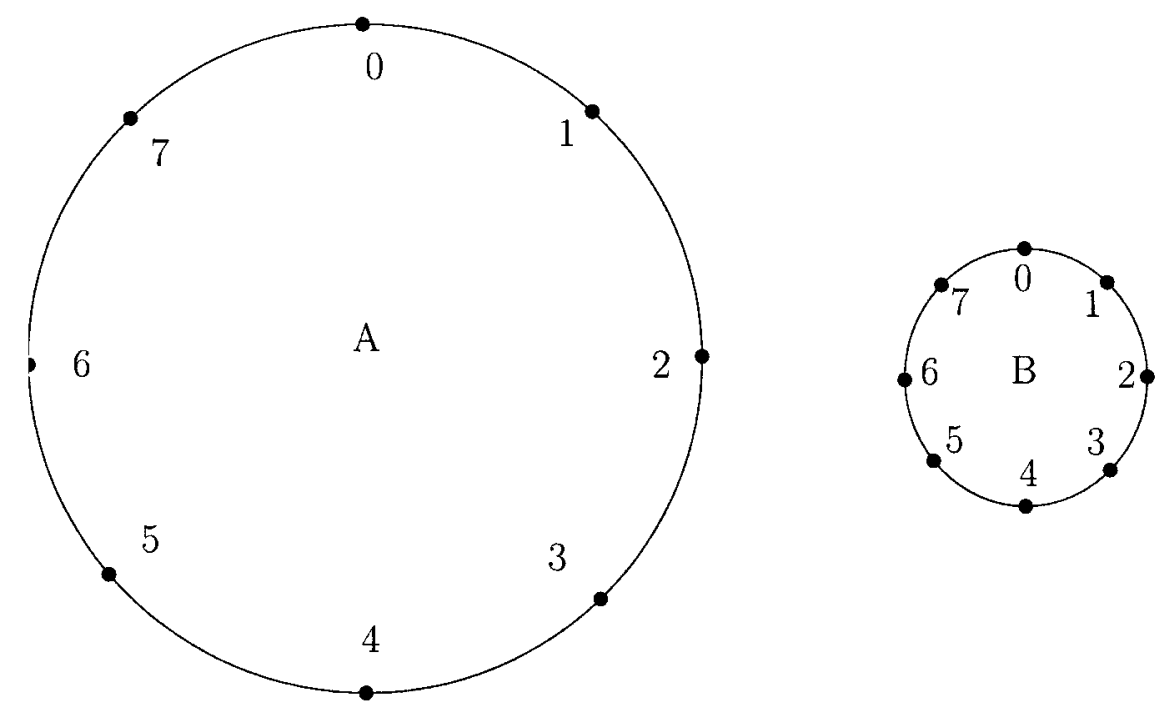

Figure 4.2: A conceptualization of the Composite code. $B$ moves clockwise until it reaches 0 , at which point $A$ moves clockwise one position also.

combined DAT.

Incrementing the last $d^{\prime}$ bits requires $\leq 6 \log d^{\prime}$ bits to be read on average and 1 bit to be written. Checking if the $d^{\prime}$-code has reached its initial state is equivalent to comparing it against a fixed bit string (that is the code's initial state). This is done an equal number of times for all possible bit strings of dimension $d^{\prime}$, and so the average number of bits read is equal to the expected position of the left-most 1 in a random bit string of dimension $d^{\prime}$ which is equal to $\frac{2^{d^{\prime}+1}-2-d^{\prime}}{2^{d^{\prime}}} \leq 2$.

If we let $d^{\prime}=\lceil\log r\rceil$, then the RPGC $B$ has dimension at least 2, from our restriction on $r$. The average read cost of the combined code to generate each bit string in the sequence becomes no more than the average cost of the $d^{\prime}$-code, plus the cost to check if the code reached its initial state, plus the cost of $d$-code divided by the length of the $d^{\prime}$-code.

Therefore, the average number of bits read by the combined code is at most

$$
\begin{aligned}
6 \log d^{\prime}+2+\frac{r}{2^{d^{\prime}}} & =6 \log \lceil\log r\rceil+2+\frac{r}{2^{\lceil\log r\rceil}} \\
& \leq 6 \log \lceil\log r\rceil+3 .
\end{aligned}
$$

In the case that both $A$ and $B$ are incremented, the bits of $B$ are equal to its 
initial state, so checking this requires reading all $d^{\prime}$ bits. This happens for all possible values in $A$, which requires reading $p$ bits in the worst case. Therefore, at some time, the new code will read $p+d^{\prime}=p+\lceil\log r\rceil$ bits.

The number of writes in the worst case, is the number of bits that must change to update both the code contained in the first $d$ bits, and the code contained in the last $d^{\prime}$ bits. Thus the number of bits written per increment operation is at most (bits written by $d$-code $)+$ (bits written by $d^{\prime}$-code $) \leq w+1$.

\subsection{RPGC-Composite Code}

We are able to use the RPGC from Theorem 4.5 with our Composite code from Lemma 4.6 to construct a new space-optimal DAT that generates a code. By applying Lemma 4.6 to the RPGC, and then repeatedly applying it $c-1$ more times to the resulting DAT, we create a DAT that generates a code while reading on average no more than $6 \log ^{(2 c-1)} d+11$ bits, and never writing more than $c$ bits to generate each bit string, for any constant $c \geq 1$.

We use the following lemmas to prove Theorem 4.10.

Lemma 4.7. Given $r \geq 16$, then $6 \log (\log r+1) \leq 6 \log \log r+2$.

Proof. Let $x=\log r$. Then we want to prove that given $x \geq 4,6 \log (x+1) \leq$ $6 \log x+2$. It is equivalent to show that $\log (1+1 / x) \leq \log (1+1 / 4) \leq 1 / 3$. Therefore $6 \log (\log r+1) \leq 6 \log \log r+2$.

Lemma 4.8. Let $c \geq 1$ and $d$ be integers such that $\log ^{(c-1)} d \geq 2$. Then $\log ^{(c)}(d / 2) \geq$ $\log ^{(c)} d-1$.

Proof. The proof is by induction. Let $c=1$. Then $\log (d / 2)=\log d-1$.

Assume the claim is true for $c$, we will show that it is then true for $c+1$. From 
our choice of $c$ and $d$, we know that

$$
\begin{aligned}
\log ^{(c)} d & \geq 2 \\
2 \log ^{(c)} d-2 & \geq \log ^{(c)} d \\
\log \left(2\left(\log ^{(c)} d-1\right)\right) & \geq \log \left(\log ^{(c)} d\right) \\
1+\log \left(\log ^{(c)} d-1\right) & \geq \log ^{(c+1)} d \\
\log \left(\log ^{(c)} d-1\right) & \geq \log ^{(c+1)} d-1 \\
\log \left(\log ^{(c)}(d / 2)\right) & \geq \log ^{(c+1)} d-1 \text { (from our inductive hypothesis) } \\
\log ^{(c+1)}(d / 2) & \geq \log ^{(c+1)} d-1,
\end{aligned}
$$

as required.

Lemma 4.9. Let $d$ and $c \geq 1$ be integers such that $\log ^{(2 c+1)} d \geq 11$, and let $m=$ $d-\left\lceil\log \left(6 \log ^{(2 c-1)} d+11\right)\right\rceil$. Then $\log ^{(2 c-1)} m \geq 11$.

Proof. Given that $\log ^{(2 c+1)} d \geq 11$, then we can say that $\log \log d+4 \leq d / 2$ because $d \geq 16$. By our choice of $d$, we know that $\log d \geq 11$ and thus $d \geq 2^{11} \geq 16$. Using this, we can show that $m \geq d / 2$ as follows:

$$
\begin{aligned}
m & =d-\left\lceil\log \left(6 \log ^{(2 c-1)} d+11\right)\right\rceil \\
& \geq d-\log \left(6 \log ^{(2 c-1)} d+11\right)-1 \\
& \geq d-\log \left(6 \log ^{(2 c-1)} d+\log ^{(2 c-1)} d\right)-1 \\
& =d-\log \left(7 \log ^{(2 c-1)} d\right)-1 \\
& =d-\log ^{(2 c)} d-\log 7-1 \\
& \geq d-\left(\log ^{(2 c)} d+4\right) \\
& \geq d-(\log \log d+4) \\
& \geq d / 2 .
\end{aligned}
$$

By our choice of $c$ and $d$ we know that $\log ^{(2 c-1)} d=\log \left(\log ^{(2 c-2)} d\right) \geq 11$ and $\log ^{(2 c-2)} d \geq 2^{11} \geq 2$. Then, since $m \geq d / 2$, and with Lemma 4.8, it follows that $\log ^{(2 c-1)} m \geq \log ^{(2 c-1)}(d / 2) \geq \log ^{(2 c-1)} d-1 \geq 2^{2^{11}}-1 \geq 11$. 
Theorem 4.10. Given integers $d$ and $c \geq 1$, such that $\log ^{(2 c-1)} d \geq 11$. There exists a DAT of dimension d that generates a code of length $L=2^{d}$, where generating the next bit string requires reading on average no more than $6 \log ^{(2 c-1)} d+11$ bits and writing in the worst case at most $c$ bits.

Proof. The proof is by induction on $c$. Let $c=1$. In this case, the Recursive Partition Gray Code in Section 4.1 satisfies the requirements. From our choice of $d$, the RPGC will have dimension at least two. It requires reading no more than $6 \log d \leq 6 \log ^{(1)} d+11$ bits on average, and writes 1 bit in each generating step.

Then let $c \geq 1$, and assume that the theorem is true for $c$. We will show it is true for $c+1$.

Let $d$ be such that $\log ^{(2 c+1)} d \geq 11$, and define $m=d-\left\lceil\log \left(6 \log ^{(2 c-1)} d+11\right)\right\rceil$. Then from Lemma 4.9 we know that $\log ^{(2 c-1)} m \geq 11$. Thus we assume our initial claim holds true for $c$, and there exists a DAT $M$ of dimension $m$ that requires reading on average no more than $6 \log ^{(2 c-1)} m+11 \leq 6 \log ^{(2 c-1)} d+11$ bits, and writing at most $c$ bits for each transition.

We use the construction from Lemma 4.6 to perform the inductive step and construct a new DAT. As input to Lemma 4.6, we use our DAT $M$, which gives us $r=6 \log ^{(2 c-1)} d+11 \geq 3$ and $w=c$. This produces a new composite DAT of dimension $m+\lceil\log r\rceil=m+\left\lceil\log \left(6 \log ^{(2 c-1)} d+11\right)\right\rceil=d$ that reads no more than $6 \log \lceil\log r\rceil+3$ bits. Note that from our choice of $c$ and $d, \log 7 \leq \log 11 \leq \log ^{(2 c)} d$. 
Then our new DAT will read on average no more than

$$
\begin{aligned}
6 \log [\log r\rceil+3 & \leq 6 \log (\log r+1)+3 \\
& \leq 6 \log \log r+5(\text { from Lemma } 4.7) \\
& =6 \log \log \left(6 \log ^{(2 c-1)} d+11\right)+5 \\
& \leq 6 \log \log \left(6 \log ^{(2 c-1)} d+\log ^{(2 c-1)} d\right)+5 \\
& =6 \log \log \left(7 \log ^{(2 c-1)} d\right)+5 \\
& =6 \log \left(\log 7+\log ^{(2 c)} d\right)+5 \\
& \leq 6 \log \left(\log { }^{(2 c)} d+\log ^{(2 c)} d\right)+5 \\
& =6 \log \left(2 \log ^{(2 c)} d\right)+5 \\
& \left.=6 \log 2+6 \log ^{(2 c+1)} d\right)+5 \\
& \left.=6 \log { }^{(2 c+1)} d\right)+11
\end{aligned}
$$

bits, as required.

From Lemma 4.6, in the worst case, our DAT writes at most $w+1=c+1$ bits to generate the next bit string. These satisfy our initial claim, finishing the proof.

Thus, there exists a DAT for any $c \geq 1$, when $\log ^{(2 c-1)} d \geq 11$, that generates all $2^{d}$ bit strings of a code of dimension $d$, and requires reading on average no more than $6 \log ^{(2 c-1)} d+11$ bits, and writing at most $c$ bits to generate each successive bit string from the previous string.

\subsection{Reading a constant average number of bits}

We have shown in Section 4.3 that it is possible to construct a code that requires reading, on average, at most $6 \log ^{(2 c-1)} d+11$ bits, and never requires writing more than $c$ bits, for any constant $c \geq 1$. This allows reading a small number of bits while writing at most a constant amount.

From Theorem 4.10, by taking $c$ to be a function of $\log ^{*} d$, it immediately follows that we can create a DAT that generates all $2^{d}$ bit strings of dimension $d$, for which 
each generating step requires reading a constant number of bits on average. This is a trade off, as the DAT requires writing at most $O\left(\log ^{*} d\right)$ bits in the worst case, meaning the code generated by this DAT is not considered a quasi-Gray code.

Corollary 4.11. There exists a space-optimal DAT of dimension d which has the following properties for each generating step:

1. For any $d>2^{16}$, the DAT reads no more than $6 \cdot 2^{2^{16}}+11$ bits on average, and writes no more than $\left\lfloor\left(\log ^{*} d-1\right) / 2\right\rfloor$ bits in the worst case.

2. For any $d>3+2^{17}$, the DAT reads no more than 100 bits on average, and writes no more than $\left\lfloor\left(\log ^{*} d+1\right) / 2\right\rfloor$ bits in the worst case.

3. For any $d>10+2^{17}$, the DAT reads no more than 20 bits on average, and writes no more than $\left\lfloor\left(\log ^{*} d+3\right) / 2\right\rfloor$ bits in the worst case.

4. For any $d>15+2^{17}$, the DAT reads no more than 17 bits on average, and writes no more than $\left\lfloor\left(\log ^{*} d+5\right) / 2\right\rfloor$ bits in the worst case.

Proof. Let $d>2^{16}$, and $c=\left\lfloor\left(\log ^{*} d-3\right) / 2\right\rfloor$. Then $c \geq 1$ and $\log ^{(2 c-1)} d \geq 11$ and it follows from Theorem 4.10 that there exists a space-optimal DAT of dimension $d$, which has the following properties for each generating step: In the average case, it requires reading no more than $6 \log ^{\left(2\left\lfloor\left(\log ^{*} d-3\right) / 2\right\rfloor-1\right)} d+11 \leq 6 \log ^{\left(2\left(\left(\log ^{*} d-3\right) / 2-1 / 2\right)-1\right)} d+$ $11 \leq 6 \log ^{\left(\log ^{*} d-5\right)} d+11 \leq 6 \cdot 2^{2^{16}}+11$ bits. And in the worst case, it requires writing at most $\left\lfloor\left(\log ^{*} d-1\right) / 2\right\rfloor$ bits.

Let $d>3+2^{17}$, and $m=d-\left(3+2^{16}\right)>2^{16}$. From our previous statement, we can construct a DAT of dimension $m$, which requires reading at most $6 \cdot 2^{2^{16}}+11 \leq 2^{3+2^{16}}$ bits on average, and writing no more than $\left\lfloor\left(\log ^{*} m-1\right) / 2\right\rfloor \leq\left\lfloor\left(\log ^{*} d-1\right) / 2\right\rfloor$ bits in the worst case. Use this DAT with Lemma 4.6, setting $r=2^{3+2^{16}}$ and $w=\left\lfloor\left(\log ^{*} d-1\right) / 2\right\rfloor$. Then there exists a DAT of dimension $m+\lceil\log r\rceil=m+3+2^{16}=d$, that requires reading on average no more than $6 \log \lceil\log r\rceil+3 \leq 100$ bits to generate the next bit string in the code, and writes no more than $\left\lfloor\left(\log ^{*} d+1\right) / 2\right\rfloor$ bits in the worst case.

Let $d>10+2^{17}$, and $m=d-7>3+2^{17}$. Use the DAT from our previous statement with Lemma 4.6, setting $r=100$ and $w=\left\lfloor\left(\log ^{*} d+1\right) / 2\right\rfloor$. Then there 
exists a DAT of dimension $m+\lceil\log r\rceil=m+7=d$, which requires reading on average no more than $6 \log \lceil\log r\rceil+3 \leq 20$ bits to generate the next bit string in the code, and writes no more than $\left\lfloor\left(\log ^{*} d+3\right) / 2\right\rfloor$ bits in the worst case.

Let $d>15+2^{17}$, and $m=d-5>10+2^{17}$. Use the DAT from our previous statement with Lemma 4.6, setting $r=20$ and $w=\left\lfloor\left(\log ^{*} d+3\right) / 2\right\rfloor$. Then there exists a DAT of dimension $m+\lceil\log r\rceil=m+5=d$, which requires reading on average no more than $6 \log \lceil\log r\rceil+3 \leq 17$ bits to generate the next bit string in the code, and writes no more than $\left\lfloor\left(\log ^{*} d+5\right) / 2\right\rfloor$ bits in the worst case.

\subsection{Lazy counters}

A lazy counter is a structure for generating a sequence of bit strings. In the first $n$ bits, it counts through the standard binary representations of 0 to $2^{n}-1$. However, this can require updating up to $n$ bits, so an additional data structure is added to slow down these updates, making it so that each successive state requires fewer bit changes to be reached. We present a few known lazy counters, and then improve upon them, using our results to generate quasi-Gray codes.

\subsubsection{LazyIncrement}

Frandsen et al. [SFMS97] describe a lazy counter of dimension $d$ that reads and writes at most $O(\log d)$ bits for an increment operation. The algorithm uses $d=n+\log n$ bits, where the first $n$ are referred to as $b$, and the $\operatorname{last} \log n$ are referred to as $i$. A state in this counter is the concatenation of $b$ and $i$, thus each state is a bit string of dimension $d$. In the initial state, all bits in $b$ and $i$ are set to 0 . The counter then moves through $2^{n+1}-2$ states before cycling back to the initial state, generating a cyclic code.

The bits of $b$ move through the standard binary numbers. However, moving from one such number to the next may require writing as many as $n$ bits. The value in $i$ is a pointer into $b$. For a standard binary encoding, the algorithm to move from one number to the next is as follows: starting at the right-most (least significant) bit, for 
each 1 bit, flip it to a 0 and move left. When a 0 bit is found, flip it to a 1 and stop. Thus the number of bit flips required to reach the next standard binary number is equal to one plus the position of the right-most 0 . This counter simply uses $i$ as a pointer into $b$ such that it can flip a single 1 to a 0 each increment step until $i$ points to a 0 , at which point it flips the 0 to a 1 , resets $i$ to 0 , and $b$ has then reached the next standard binary number. The algorithm is stated as follows:

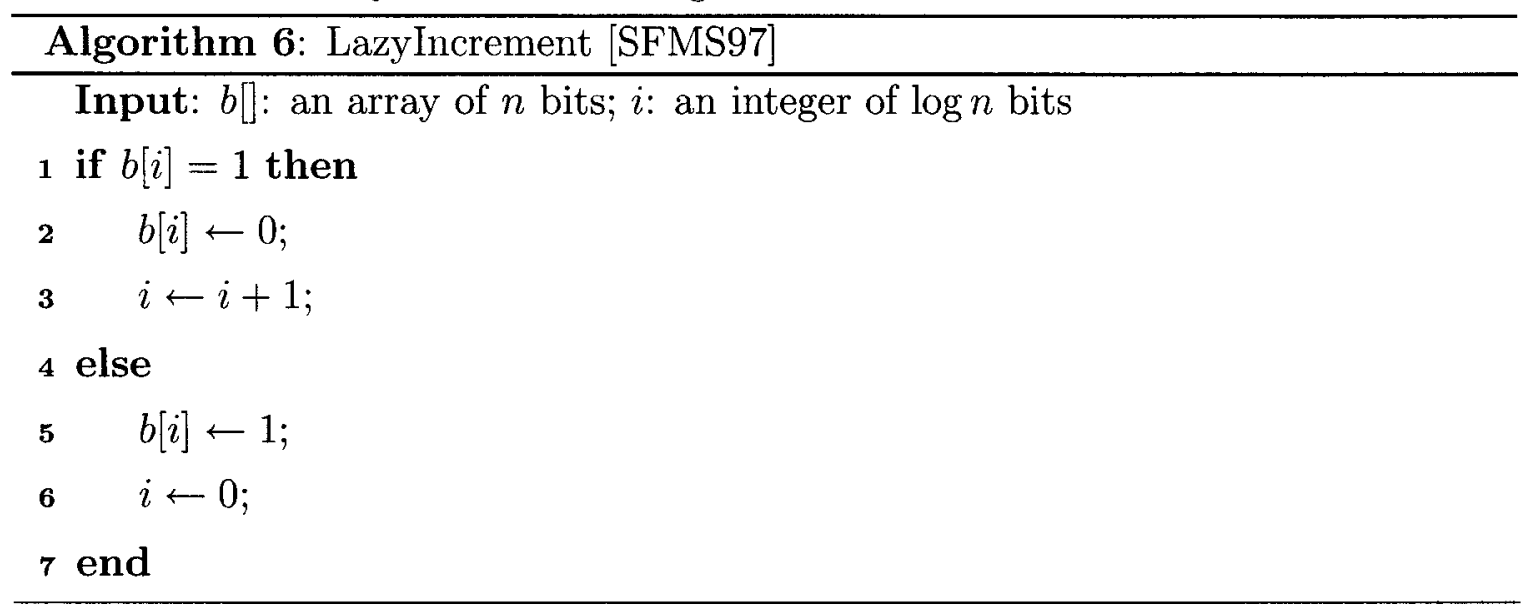

Lemma 4.12. [SFMS97] Let $n \geq 2$ be a power of two. There exists a DAT of dimension $d=n+\log n$, using the Lazy Increment algorithm, that generates $2^{n+1}-2$ of a possible $n 2^{n}$ bit strings, where in the limit $n \rightarrow \infty$ the space efficiency drops to 0 . The DAT reads and writes in the worst case $\log n+1$ bits to generate each successive bit string, and on average reads and writes 3 bits.

Proof. The maximum number of bit strings generated by this counter is $2^{d}=n 2^{n}$, however it actually generates significantly less.

The bits of $b$ move through each standard binary number, but with additional states in between, such that each state in differs by a single bit in $b$. Thus, the number of states between two of these numbers is equal to the number of the bits that need to change, and this is equal to the distance to the right-most 0 bit in $b$.

The right-most bit in $b$ is 0 for half of the standard binary numbers. For the other half, there is a 0 to the left of it half of the time. If the right-most bit is position 1 , then bit $j$ contains the right-most 0 exactly $2^{n} / 2^{j}$ times, over all standard binary numbers which fit in $n$ bits. Meanwhile, the bits in $i$ make it possible to have these 
transitional states, but don't provide any additional states beyond them. Thus we can count the number of total states in the counter. Bit $j$ is the right-most 0 in $b$ for $2^{n} / 2^{j}$ numbers, and each time it is, it requires $j$ bit flips to reach the next number. Additionally, when there are no 0 bits in $b$ at all, $n$ bit flips are required to reach the next number, that returns $b$ back to all zeros. Let $G(d)$ be the number of bit strings generated by LazyIncrement, for dimension $d=n+\log n$. Then the number of generated bit strings is $G(d)=\sum_{j=1}^{n} j \frac{2^{n}}{2^{j}}+n=2^{n} \sum_{j=1}^{n} \frac{j}{2^{j}}+n=2^{n+1}-2$.

The space efficiency of this counter, or the ratio of bit strings generated to the number of possible strings is $\frac{G(d)}{2^{d}}=\frac{2^{n+1}-2}{2^{n+\log n}}=\frac{2^{n}-1}{n 2^{n-1}}$. Since $\lim _{n \rightarrow \infty} \frac{2^{n}-1}{n 2^{n-1}}=0$, the counter is non-space-optimal, and the space efficiency of the counter grows worse as its dimension grows larger.

To generate each successive bit string, the worst-case number of bits read or written by this counter is the cost to increment or reset $i$, which is an integer of dimension $\log n$, plus the single bit which changes in $b$. Thus the counter never reads nor writes more than $\log n+1$ bits. On average, incrementing an integer (using the standard binary representation) requires reading and writing at most 2 bits, while only a single bit is ever read or written in $b$ at a time. Thus the average cost to generate a bit string in this counter is at most 3 .

The LazyIncrement algorithm presented above can be constructed as DAT. First construct a DAT that reads the value of $i$. This tree reads all $\log n$ bits of $i$ and has $n$ leaves, one for each value of $i$. The leaf that represents $i=x$ will then read bit $x$ of $b$. If the bit was a 0 , it is changed to a 1 , and all of the bits in $i$ are set to 0 . If the bit was a 1 , then the bit is changed to a 0 and $i$ is changed to represent $x+1$.

\subsubsection{SpinIncrement}

An observation by Brodal [Bro09] (unpublished) leads to a dramatic improvement in space efficiency over the previous algorithm by adding a single bit to the counter. This extra bit allows for the $\log n$ bits in $i$ to spin through all their possible values, thus making better use of the bits and generating more bit strings with them. The variables $b$ and $i$ are unchanged from the counter in Lemma 4.12, and $k$ is a single bit, 
making the counter have dimension $d=n+\log n+1$. The algorithm is as follows.

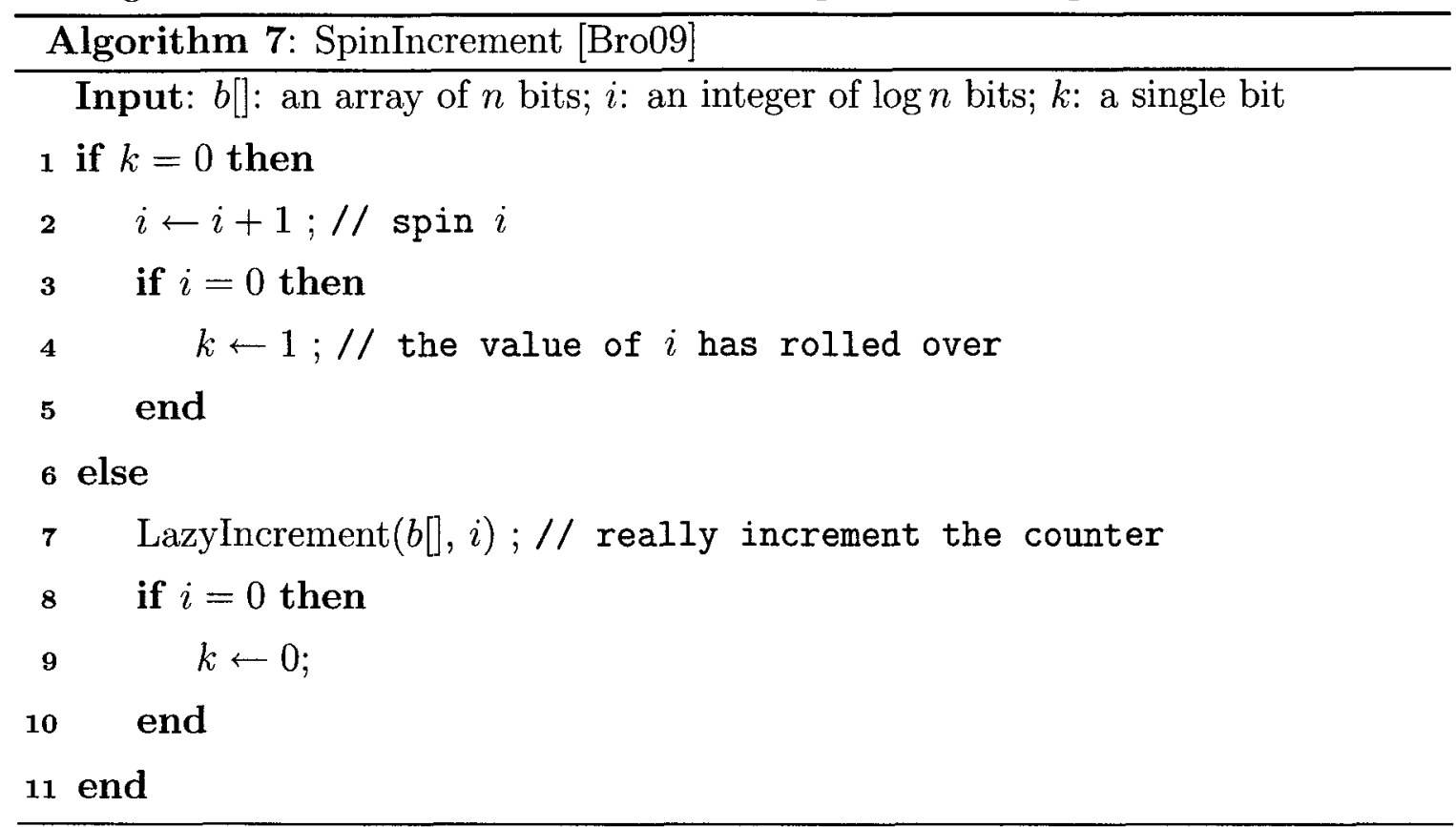

Lemma 4.13. [Bro09] Let $n \geq 2$ be a power of two. There exists a DAT of dimension $d=n+\log n$, using the SpinIncrement algorithm, that generates $(n+1)\left(2^{n}-1\right)$ of a possible $2 n 2^{n}$ bit strings, where in the limit $n \rightarrow \infty$ the space efficiency converges to $1 / 2$. The DAT reads and writes in the worst case $\log n+2$ bits to generate each successive bit string, and on average reads at most 4 bits.

Proof. This counter spins through $i$ every time that $b$ becomes equal to its next value in the standard binary representation, for which there are $2^{n}$ such cases. Spinning through all values for $i$ adds exactly $2^{\log n}=n$ states. Thus the total number of states for this improved lazy counter is $n 2^{n}+2^{n+1}-2-2^{n}=n 2^{n}+2^{n}-2=(n+1) 2^{n}-2$.

The space efficiency of this improved counter is $\frac{(n+1) 2^{n}-2}{2 n 2^{n}}$. In the limit $n \rightarrow \infty$ the space efficiency converges to $1 / 2$. That is, when the counter has large dimension $d$, approximately half of the $2^{d}$ possible bit strings are generated.

The worst-case number of bits read or written by this counter is one more than the counter in Lemma 4.12. The only cases added are where $i$ and $k$ are changed, which together do not exceed that bound. However, in the case where $b$ and $i$ are changed, $k$ may also be read and changed, so the counter may read and write at most $\log n+2$ 
bits to generate the next bit string. Checking the value of $k$ requires reading a single bit, incrementing $i$ requires on average to read and write at most 2 bits. Comparing $i$ for equality to the fixed bit string $000 \ldots 0$ can be done for free after incrementing $i$ in the DAT model. The LazyIncrement algorithm reads at most 3 bits on average, after $k$ has been read, and is run an average of 2 times with $n$ increments in between, contributing at most 4 to the average. From these observations, it follows that the average number of bits read and written by this counter, to generate the next bit string, does not exceed 4.

The SpinIncrement algorithm can be constructed as DAT using the DAT from Lemma 4.12. Add a new root node that reads $k$. When $k=0$, go to its left child. The right child is a subtree that reads all the bits of $i$. For all leaf nodes of this subtree, $i$ is incremented. And when $i$ was equal to $n-1, k$ is also set to 0 . When $k=1$, go to its right child, which is the root of a subtree mostly identical to the DAT for Lazy Increment, with an extra rule in the leaf where $i$ is set to $000 \ldots 0$, that sets $k$ to 0 as well.

\subsubsection{DoubleSpinIncrement}

By generalizing the dimension of $k$, we are able to make the counter even more space efficient while keeping its $O(\log n)=O(\log d)$ worst-case bound for bits written and read. Let $k$ be a bit array of dimension $g \geq 1$. Then for a counter of dimension $d=n+\log n+g$, the new algorithm is as follows. 


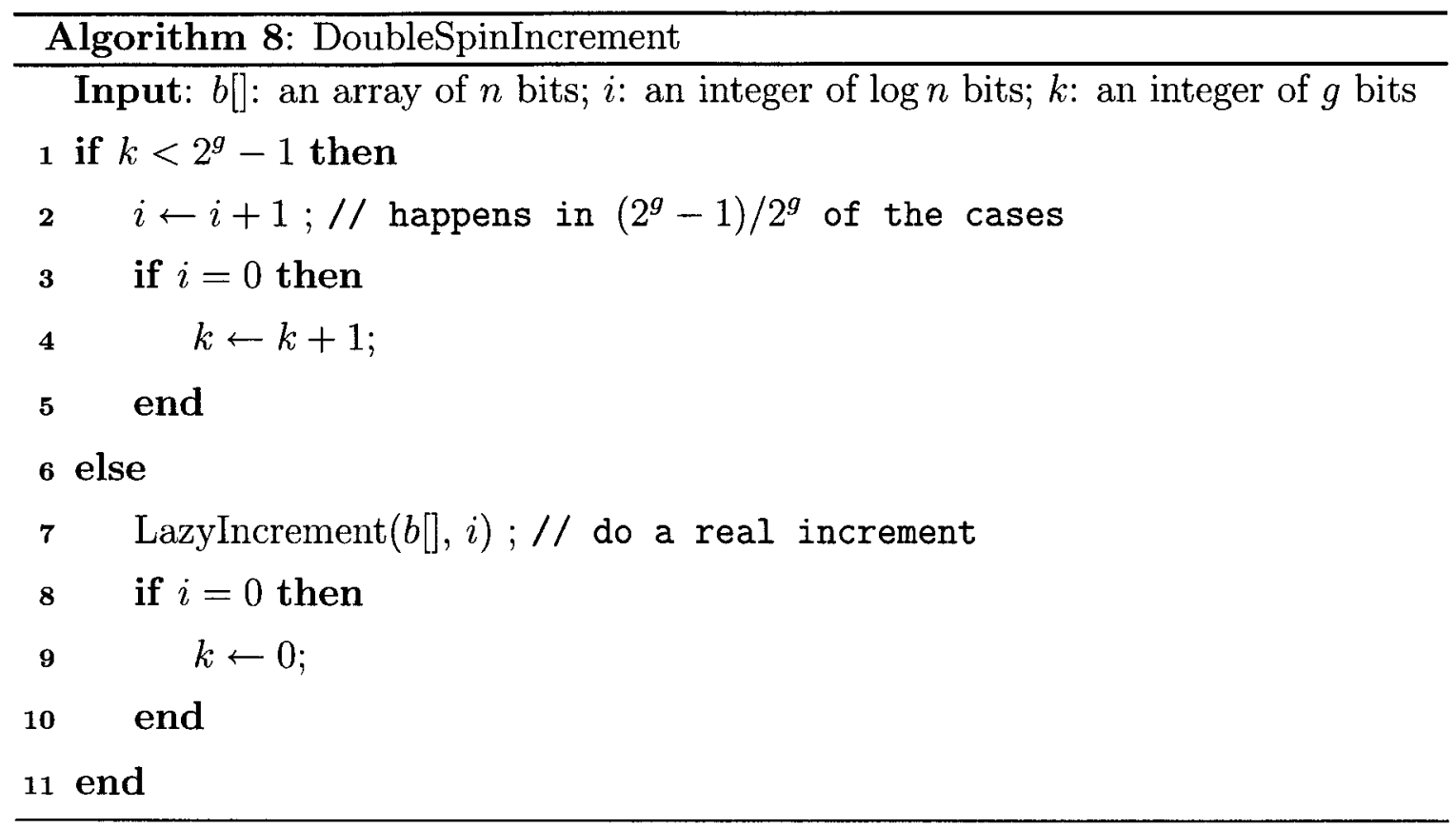

Theorem 4.14. Let $n \geq 2$ be a power of two, and $g \geq 1$ be an integer. There exists a $D A T$ of dimension $d=n+\log n+g$ with space efficiency $1-O\left(2^{-g}\right)$. The DAT, using the DoubleSpinIncrement algorithm, reads and writes in the worst case $g+\log n+1$ bits to generate each successive bit string, and on average reads and writes $O(1)$ bits. Proof. This counter generates $2^{g}-1$ states for each time it spins through the possible values of $i$. Thus the number of bit strings generated is $n 2^{n}\left(2^{g}-1\right)+2^{n}-2=$ $n 2^{n} 2^{g}-(n-1) 2^{n}-2$. Given the dimension of the counter, the possible number of bit strings generated is $n 2^{n} 2^{g}$. This gives a space efficiency of $1-O\left(2^{-g}\right)$. When $g=1$, we have exactly the same counter as given by Lemma 4.13, and when $g$ is an increasing function of $n$, we produce a counter with space efficiency arbitrarily close to one.

In the worst case, this counter reads and writes every bit in $i$ and $k$, and a single bit in $b$, thus $g+\log n+1$ bits. On average, the counter now reads and writes $O(1)$ bits. This follows from a similar argument to that made for Lemma 4.13, where each line modified in the algorithm still reads on average $O(1)$ bits.

The DoubleSpinIncrement can also be constructed as DAT by building on the DAT from Lemma 4.13. Replace the root node with a subtree that reads all the bits 
of $k$ and has $2^{g}$ leaf nodes. For leaf nodes that read a value in $k$ less than $2^{g}-1$, the leaf node becomes the root of a subtree similar to the right child of the root node in the DAT for SpinIncrement. These subtrees are modified in that $k$ is incremented instead of set to 0 when $i$ was equal to $n-1$. The one leaf node where $k=2^{g}-1$ becomes the root of a subtree identical to the left child of the root node in the DAT for SpinIncrement.

Corollary 4.15. Let $n \geq 2$ be a power of two, and $g=t \log n$ be an integer, for $t>0$. There exists a DAT of dimension $d=n+(t+1) \log n$ with space efficiency $1-O\left(n^{-t}\right)$. The DAT, using the DoubleSpinIncrement algorithm, reads and writes in the worst case $(t+1) \log n+1$ bits to generate each successive bit string, and on average reads and writes $O(1)$ bits.

Proof. This follows from Theorem 4.14 by substituting $g=t \log n$.

\subsubsection{WineIncrement}

Rahman and Munro [RM08] present a counter that reads at most $\log n+4$ bits and writes at most 4 bits to perform an increment or decrement operation. The counter uses $n+1$ bits to count through $2^{n}$ states, and has space efficiency $2^{n} / 2^{n+1}=1 / 2$. Compared to DoubleSpinIncrement, their counter writes fewer bits per generating step, but is less space efficient. By modifying our lazy counter to use Gray codes internally, the worst-case number of bits read remains asymptotically equivalent to the counter by Rahman and Munro, and the average number of bits we read increases. We are able to write a smaller constant number of bits per increment and retain a space efficiency arbitrarily close to 1 .

We modify our counter in Theorem 4.14 to make $i$ and $k$ hold a cyclic Gray code instead of a standard binary number. The BRGC is a suitable Gray code for this purpose, so we will use it. Given a function next $(j)$ that takes a bit string $j$ of rank $r$ in the BRGC and returns the bit string of rank $r+1$, and a function $\operatorname{rank}(j)$ that returns the rank value of the bit string $j$ in the BRGC. The following algorithm provides a lazy counter of dimension $d=n+\log n+g$, where $g \geq 1$, that writes at 
most 3 bits, and reads at most $g+\log n+1$ bits to generate the next state, and is space-optimal in the limit $n \rightarrow \infty$.

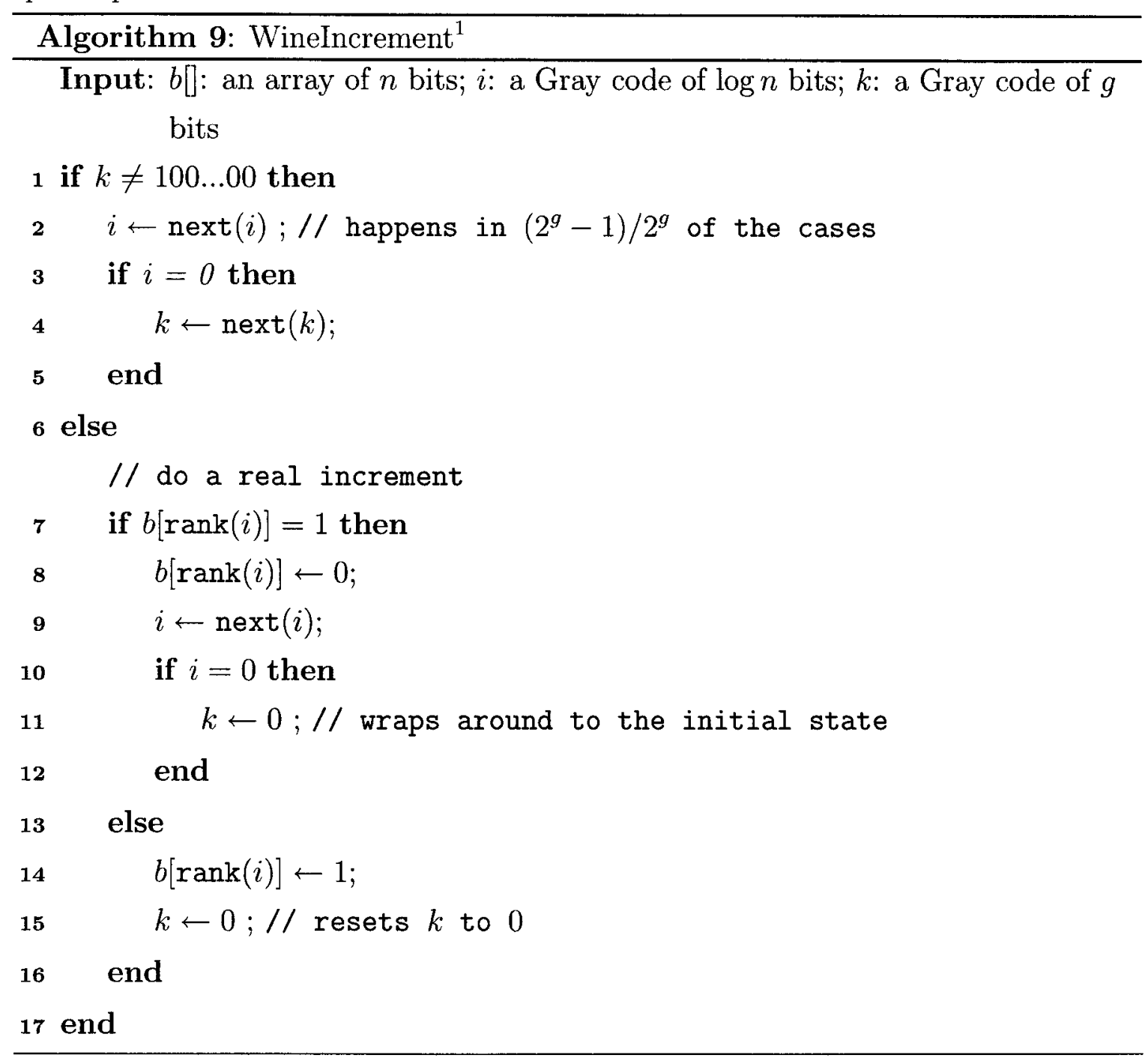

Theorem 4.16. Let $n \geq 2$ be a power of two, and $g \geq 1$ be an integer. There exists a DAT of dimension $d=n+\log n+g$ with space efficiency $1-O\left(2^{-g}\right)$. The DAT, using the WineIncrement algorithm, reads in the worst case $g+\log n+1$ bits and writes in the worst case 3 bits to generate each successive bit string.

\footnotetext{
${ }^{1}$ The name WineIncrement comes from the Caribbean dance known as Wineing, a dance that is centered on rotating your hips with the music. The dance is pervasive in Caribbean culture, and has been popularized elsewhere through songs and music videos such as Alison Hinds' "Roll It Gal", Destra Garcia's "I Dare You", and Fay-Ann Lyons" "Start Wineing".
} 
Proof. The number of states used by this counter would be the same as the DoubleSpinIncrement algorithm, except that we are unable to reset $i$ to 0 when a bit in $b$ is flipped to 1 without changing up to $\log n$ bits in $i$. Instead, we leave $i$ as it is and observe that it does not significantly reduce the space efficiency of the counter.

Note that when a bit in $b$ is flipped to a 1 :

- $\operatorname{rank}(i)$ points to the index of the right-most 1 in $b$, counting from the leastsignificant bit.

- a bit in $b$ flips to 1 if and only if the bit sequence $b$ is entering the next state of the standard binary number encoding. That is, $b$ counts from 0 thru $2^{n}-1$, and back to 0 , as a standard binary number, with extra states in between. The extra states all come from steps where a bit in $b$ flips to 0 .

Based on these observations we can sum up all the values of $i$ that occur when a bit in $b$ flips to 1 as the positions of the right-most 1 in all possible bit strings of dimension $n$, which is $2^{n} \sum_{i=1}^{n} \frac{i}{2^{i}}=2^{n+1}-n-2$. When $i$ has a value greater than 0 , the number of states lost compared to DoubleSpinIncrement is exactly the difference between $i$ and 0 . Thus, over all increment steps, this summation describes the total number of states lost compared to our third lazy counter. Therefore the total number of states used is $n 2^{n} 2^{g}-(n-1) 2^{n}-2-2^{n+1}+n+2=n 2^{n} 2^{g}-(n+1) 2^{n}+n$. The number of bits, and thus, the number of possible states, is unchanged from the DoubleSpinIncrement algorithm. There are $2^{n+\log n+g}$ possible states, and the space efficiency of this counter as $n$ grows large converges to

$$
\lim _{n \rightarrow \infty} \frac{n 2^{n} 2^{g}-(n+1) 2^{n}+n}{n 2^{n} 2^{g}}=1-\lim _{n \rightarrow \infty} \frac{(n+1) 2^{n}-n}{n 2^{n} 2^{g}}=1-O\left(2^{-g}\right) .
$$

The average number of bits read for each line of the algorithm is $O(1)$, with the exception of the lines which increment $i$ and $k$. When they are executed, these lines read on average $\log n$ and $g$ bits respectively, so the total average number of bits read is at most $\log n+g+1$.

The counter writes at most one bit in each of $b, i$, and $k$, and thus writes in the worst case 3 bits per increment operation. 
Corollary 4.17. Let $n \geq 2$ be a power of two, and $t \log n$ be an integer, for $t>0$. There exists a DAT of dimension $d=n+(t+1) \log n$ with space efficiency $1-O\left(n^{-t}\right)$. The DAT, using the WineIncrement algorithm, reads in the worst case $(t+1) \log n+1$ bits and writes in the worst case 3 bits to generate each successive bit string.

Proof. This follows from Theorem 4.16 by substituting $g=t \log n$.

From Corollary 4.17, we get a counter that is more space efficient than previously known counters, with a constant number of bits written for each increment. But while the counter reads at most $(t+1) \log n+1$ bits in the worst case, its average number of bits read is also $O(\log n)$. Using the quasi-Gray code counter from Theorem 4.10, we are able to bring the average number of bits read down as well. The worst case number of bits read remains $(t+1) \log n+1$, but on average, we only read at most $12 \log ^{(2 c)} n+O(1)$ bits, for any $c \geq 1$.

The algorithm does not need to change from its fourth iteration for these modifications. We simply make $i$ a quasi-Gray code from Theorem 4.10 of dimension $\log n$ and $k$ a similar quasi-Gray code of dimension $g$.

Theorem 4.18. Let $n$ be a power of two such that $\log ^{(2 c)} n \geq 11$ and $g$ be an integer such that $\log ^{(2 c-1)} g \geq 11$. Then for any $c \geq 1$, there exists a DAT of dimension $d=n+\log n+g$ bits, using the WineIncrement algorithm, with space efficiency $1-O\left(2^{-g}\right)$, that reads in the worst case $g+\log n+1$ bits, writes in the worst case $2 c+1$ bits, and reads on average no more than $12 \log ^{(2 c)} n+O(1)$ bits.

Proof. We determine the average number of bits read in the previous algorithm for each line of the WineIncrement function. Line 1 becomes true each time $b$ reaches a standard binary number representation, and there are $2^{n}$ such numbers. Each time the line becomes true, it stays so while $i$ and $k$ spin through their values together. During this time, the value of $k$ changes every $n$ steps, and we see all possible values of $k$ except its maximally ranked state $1000 \ldots 0$. In the DoubleSpinIncrement algorithm, this would account for $n 2^{n}\left(2^{g}-1\right)$ states, but all states lost due to $i$ happen during this stage, and so it accounts instead for $n 2^{n}\left(2^{g}-1\right)-2^{n+1}+n+2=n 2^{n} 2^{g}-(n+2) 2^{n}+n+2$ states. 
If we saw each value of $k$ once, the number of bits read to test if the string is equal to $1000 \ldots 0$ would be $\sum_{i=1}^{g} i \cdot 2^{g} / 2^{i}=2^{g+1}-g-2$. Instead, we see each value in $k$ for $n$ consecutive steps, except for state $1000 \ldots 0$. Therefore, the number of bits read between states where $k=1000 \ldots 0$ is $n\left(2^{g+1}-g-2-g 2^{g} / 2^{g}\right)=n 2^{g+1}-2 n g-2 n$.

During the remaining $2^{n}-2$ states, $k=1000 \ldots 0$, and line 1 will read all $g$ bits each time it is executed, reading a total of $g 2^{n}-2 g$ bits. Thus the the average number of bits read by line 1 is

$$
\begin{aligned}
R_{1} & =\frac{n 2^{g+1}-2 n g-2 n+g 2^{n}-2 g}{n 2^{n} 2^{g}-(n+1) 2^{n}+n} \\
& \leq \frac{n 2^{g+1}+g 2^{n}}{n 2^{n+g}-(n+1) 2^{n}+n} \\
& =\frac{1}{2^{n-1}} \frac{n 2^{n+g}}{n 2^{n+g}-(n+1) 2^{n}+n}+\frac{g}{n 2^{g}} \frac{n 2^{n+g}}{n 2^{n+g}-(n+1) 2^{n}+n} \\
& =\frac{1}{2^{n-1}} \frac{n 2^{n+g}-(n+1) 2^{n}+n+(n+1) 2^{n}-n}{n 2^{n+g}-(n+1) 2^{n}+n} \\
& +\frac{g}{n 2^{g}} \frac{n 2^{n+g}-(n+1) 2^{n}+n+(n+1) 2^{n}-n}{n 2^{n+g}-(n+1) 2^{n}+n}(n+1) 2^{n} \\
\leq & \frac{1}{2^{n-1}}\left(1+\frac{(n+1) 2^{n}}{n 2^{n+g}-(n+1) 2^{n}+n}\right)+\frac{g}{n 2^{g}}\left(1+\frac{2^{n}}{n 2^{n+g}-(n+1) 2^{n}+n}\right) \\
\leq & 2+\frac{2(n+1)}{n 2^{n+g}-(n+1) 2^{n}+n}+\frac{\left(n 2^{n+g}-(n+1) 2^{n}+n\right.}{n(1)}, \\
& =O(1)
\end{aligned}
$$

since each of the two fractions are at most one when $n \geq 2$.

Line 2 increments our counter from Theorem 4.10, and reads on average $R_{2} \leq$ $6 \log ^{(2 c-1)}(\log n)+11=6 \log ^{(2 c)} n+11$ bits.

Line 3 checks if $i$ is equal to a specific value. This check is done consecutively over all values of $i$. Thus half the time, only one bit needs to be checked, a quarter of the time, two bits, and so on. The average number of bits read for this line is then $\sum_{i=1}^{\log n} i \frac{n}{2^{i}}$, each time it is reached. Once the check in line 1 returns false, it does so once for each value of $k$ other than 10..00. Thus this line is executed $\left(2^{g}-1\right)\left(2^{n}-1\right)$ times. 
The average number of bits read by line 3 is

$$
\begin{aligned}
R_{3} & \leq\left(\sum_{i=1}^{\log n} i \frac{n}{2^{i}} \frac{2^{g}\left(2^{n}-1\right)}{n\left(2^{g}-1\right)\left(2^{n}-1\right)+2^{n}-2^{n+1}+n+1}\right. \\
& \leq \frac{2 n 2^{g}\left(2^{n}-1\right)}{n\left(2^{g}-1\right)\left(2^{n}-1\right)-2^{n}+n+1} \\
& =\frac{n 2^{g+1}\left(2^{n}-1\right)}{n\left(2^{g}-1\right)\left(2^{n}-1\right)-2^{n}} \\
& =\frac{2^{g+1}}{\left(2^{g}-1\right)-\frac{2^{n}}{n\left(2^{n}-1\right)}} \\
& \leq \frac{2^{g+1}}{\left(2^{g}-1\right)-1} \\
& =2 \frac{\left(2^{g}-2\right)+2}{\left(2^{g}-2\right)} \\
& =2+\frac{4}{\left(2^{g}-1\right)} \\
& =O(1) .
\end{aligned}
$$

Line 4 is similar to line 2, but with a slightly larger counter. It reads on average $R_{4} \leq 6 \log ^{(2 c-1)}(t \log n)+11=6 \log ^{(2 c-1)} t+6 \log ^{(2 c)} n+11$ bits, for $c \geq 1$.

Line 7 reads a single bit in $b$, and must read all $\log n$ bits in $i$ in order to determine its rank. The line of code is executed $2^{n+1}-2$ times over the entire sequence of 
transitions for the counter as a whole. Thus the average number of bits read is

$$
\begin{aligned}
R_{7} & =\frac{(1+\log n)\left(2^{n+1}-2\right)}{n\left(2^{g}-1\right)\left(2^{n}-1\right)+2^{n}-2^{n+1}+n+1} \\
& =\frac{2\left(2^{n}-1\right)+2\left(2^{n}-1\right) \log n}{n\left(2^{g}-1\right)\left(2^{n}-1\right)-2^{n}+n+1} \\
& \leq \frac{2\left(2^{n}-1\right)+2\left(2^{n}-1\right) \log n}{n\left(2^{g}-1\right)\left(2^{n}-1\right)-2^{n}} \\
& =\frac{2+2 \log n}{n\left(2^{g}-1\right)-1-\frac{1}{2^{n}-1}} \\
& \leq \frac{2+2 \log n}{n\left(2^{g}-1\right)-2} \\
& \leq 2+\frac{2 \log n-2 n\left(2^{g}-1\right)}{n\left(2^{g}-1\right)} \\
& \leq 2+\frac{2 \log n}{n\left(2^{g}-1\right)} \\
& =O(1) .
\end{aligned}
$$

Line 8 reads all $\log n$ bits of $i$, similarly to line 7 , and is executed no more times than is line 7. Thus the average number of bits read for line 8 is $R_{8} \leq O(1)$.

Line 9 has the average cost as line 2 per execution, and is executed fewer times over all generated states, thus $R_{9} \leq R_{2}$ bits.

Line 10 has an average cost per execution that is the same as line 3 , but is executed fewer times, at most $2^{n+1}-2$ times. Thus its average cost $R_{10} \leq O(1)$.

Line 11 does not need to read any bits, as the state of $k$ is already known from line 1.

Line 14 is similar to line 8 , and does not read more bits on average than line 7 . Its average cost is $R_{14} \leq 7$.

Line 15 , like line 11 , does not need to read any bits.

The total average number of bits read can be determined by the summation $\sum_{i=1}^{15} R_{i}$. It is clear that this average is at most $12 \log ^{(2 c)} n+O(1)$. The number of bits written in the worst case remains constant, though it grows slightly to $2 c+1$. Thus with $c=1$, we can read on average $O(\log \log n)$ bits, write at most 3 , and read in the 
worst case $g+\log n+1$ bits. This is accomplished while keeping the space efficiency arbitrarily close to 1 , as in the previous counter.

The WineIncrement algorithm can be constructed as a DAT as well, as long as the quasi-Gray codes used in $i$ and $k$ can be generated with a DAT. This is done by using the DAT structures for $i$ and $k$ inside the tree from Theorem 4.14. For this DAT, we are using a quasi-Gray code instead of standard integers for our counting variables. While this changes the description of the resulting DAT, the actual structure does not change dramatically. First, replace the root node with a subtree $T_{k}$ that reads all the bits of $k$ and has $n^{c}$ leaf nodes, labeled from 1 to $n^{c}$. Let leaf $j$ be reached when $k$ has rank $j$ in its quasi-Gray code. The highest rank leaf will read the last bit string from the code in $k$, and it becomes the root of another subtree, $T_{i_{n^{c}}}$. This subtree corresponds to the main else clause of the algorithm. The subtree reads $i$, and has $n$ leaves, one for each possible state of $i$. Again, let leaf $j$ be reached when $i$ has rank $j$ in its quasi-Gray code. Then, in the leaf node $j$ of $T_{i_{n^{c}}}$, the $j$-th bit of $b$ is read. If the bit was a 0 , it is changed to a 1 , and $k$ is moved ahead one state to 0 . If the bit was a 1 , then the bit is changed to a 0 and $i$ is changed to represent the state of rank $j+1$ in its quasi-Gray code, and lastly if $j$ is $n-1$, that is this leaf represents the highest rank for the quasi-Gray code in $i$, additionally move $k$ ahead one state to return it to 0 .

Other leaf nodes of $T_{k}$ also become roots of subtrees $T_{i_{u}}$ for $1 \leq u<n^{c}$, where $T_{i_{u}}$ is rooted at the $u$-th child of $T_{k}$, and is reached when $k$ has rank $u$ in its quasi-Gray code. These subtrees represent the many cases where the main if clause is true in the algorithm. The subtrees each read all of $i$ and have $n$ leaf nodes, one for each possible state of $i$. Each leaf node has a rule to modify $i$ such that its rank in its quasi-Gray code increases one. Additionally the $n$-th leaf of each subtree $T_{i_{u}}$, has a rule to modify $k$ to represent the state of rank $u+1$ in its quasi-Gray code.

Corollary 4.19. Let $n$ be a power of two such that $\log ^{(2 c)} n \geq 11$ and $t \log n$ be an integer, for $t>0$ such that $\log ^{(2 c-1)}(t \log n) \geq 11$. Then for any $c \geq 1$, there exists a $D A T$ of dimension $d=n+(t+1) \log n$ bits, using the WineIncrement algorithm, with space efficiency $1-O\left(n^{-t}\right)$, that reads in the worst case $(t+1) \log n+1$ bits, writes in the worst case $2 c+1$ bits, and reads on average no more than $12 \log ^{(2 c)} n+O(1)$ 
bits.

Proof. This follows from Theorem 4.18 by substituting $g=t \log n$.

Corollary 4.20. Let $n>2^{2^{16}}$ be a power of two, and $t \log n$ be an integer, for $t>0$ such that $\log ^{(2 c-1)}(t \log n) \geq 11$. Then there exists a DAT of dimension $d=$ $n+(t+1) \log n$ bits, with space efficiency $1-O\left(n^{-t}\right)$, that reads in the worst case $(t+1) \log n+1$ bits, writes in the worst case $\log ^{*} n-3$ bits, and reads on average $O(1)$ bits.

Proof. Let $c=\left\lfloor\left(\log ^{*} n-4\right) / 2\right\rfloor$. By our choice of $c$ and $n$, we know $c \geq 1$ and $\log ^{(2 c)} n \geq \log ^{\left(\log ^{*} n-4\right)} n \geq 11$. Then the result follows directly from Corollary 4.19. 


\section{Chapter 5}

\section{Conclusion}

\subsection{Summary}

We have shown in this thesis how to generate a Gray code, while reading significantly fewer bits on average than previously known algorithms, and how to efficiently generate a quasi-Gray code with the same worst-case performance and improved space efficiency. We give a tradeoff between space-efficiency, and the worst-case number of bits written. When the worst case number of bits is equal to the dimension of the code, $d$, it is easy to generate all $2^{d}$ bit strings. Our Recursive Partition Gray Code gives a $d$-dimensional Gray code, writing at most a single bit to generate each successive bit string, while reading no more than $6 \log d$ bits on average and with an optimal space efficiency of 1 . As with all known algorithms that generate a Gray code, this requires all $d$ bits are read in the worst case.

But when the worst-case number of bits read decreases, our space-efficiency does also. As an improvement on previous results, we present algorithms that are able to retain space efficiency arbitrarily close to 1 by reading more than a constant number of bits on average. Our WineIncrement algorithm gives an algorithm to generate a quasi-Gray code with dimension $d=n+(t+1) \log n$. This algorithm reads on average $12 \log ^{(2 c)} n+O(1)$ bits, while in the worst case it reads only $(t+1) \log n+1$ bits. This is done with a space efficiency of $1-O\left(n^{-t}\right)$. The quasi-Gray code changes in at most $2 c+1$ bits to reach any successive state in the code. 
This trade-off highlights the initial problem which motivated this work: a lower bound on the number of bits read in the worst case, for a Gray code with space efficiency of 1 . Fredman [Fre78] showed a lower bound of $\Omega(\log d)$ bits read in the worst case, and improving this bound remains an open problem.

During our efforts to find a better lower bound, we used extensively the idea of a Gray code being equivalent to a Hamiltonian path (or cycle, for a cyclic code) on a hypercube [Lei92]. We aimed to find an example of a Gray code of dimension $d$, which read at most $d-1$ bits in the worst case, or alternatively to show that such a code is not possible. When traversing a hypercube, a rule is a directed edge between two points on the hypercube, $x$ and $y$, with the point $x$ corresponding to a path in the DAT. If every path in the DAT has length $d-1$, then it would be used in exactly two situations: when the bit it did not read is either a 0 or a 1 . In the hypercube, this means that any rule we add, from $x$ to $y$, must be paired with a second parallel rule starting from a position one hop away from $x$ and ending one hop away from $y$. Thus all rules must come in pairs, travelling parallel to each other, in the same direction, and adjacent to a shared face on the hypercube. Figure 5.1 shows an example of a hypercube with dimension three, and paired rules along its edges. Note that only one rule may exist on any one edge of the hypercube.

This model is weaker than the DAT model, as not all arrangements of rules on the hypercube may be constructed as a DAT, however all DATs of height $d-1$ can be converted to pairs of rules on the hypercube. Thus, showing such a construction is not possible on the hypercube would also show it is not possible for a DAT. If paired rules, such as those in Figure 5.1, cannot be placed without overlapping such that a Hamiltonian (cycle) path is formed on the hypercube, then it is also not possible to construct a DAT that generates a (cyclic) Gray code of dimension $d$ without reading $d$ bits in the worst case. For small $d$, it quickly becomes obvious that such pairs of rules cannot be used to construct a Hamiltonian cycle on the hypercube. For larger dimensions, we conjecture that it is never possible to construct a Hamiltonian cycle using pairs of parallel face-adjacent rules on the hypercube. 


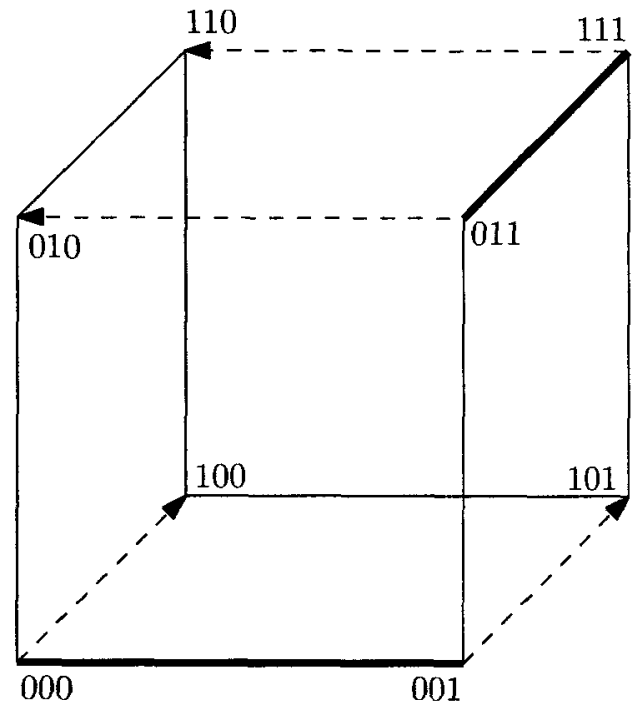

Figure 5.1: Rules for traversing a hypercube of dimension $d=3$ that require reading $d-1$ bits. The rules are directed dashed lines, each of which shows the bit that would be changed when the code's current bit string is equal to the label of the rule's source vertex in the hypercube. A dark line connects two states that differ only in the bit not read for the two paired rules adjacent to it. 


\subsection{Future work}

The main open problem remains: finding a better lower bound for the worst-case number of bits read by a Gray code with space efficiency of 1 , or finding an example of a such Gray code that always reads less than $d$ bits to generate the next bit string in the code.

When considering the average number of bits read, is it possible to find a Gray code with space efficiency 1 that requires reading less bits than the Recursive Partition Gray Code? As a Gray code, what properties does the RPGC have, such as run length, balance of bit flips, and others discussed by Savage? [Sav97]

What other properties does the Recursive Partition Gray Code have, such as Is it possible to find a quasi-Gray code with space efficiency 1 , or a quasi-Gray code that reads fewer bits on average or in the worst case than the WineIncrement algorithm? Is there a lower bound on the space efficiency in relation to the number of bits read on average? Fredman [Fre78] showed a tradeoff between the number of bits written and read in the worst case. Can a tighter tradeoff be shown, between the worst-case number of bits written, the worst-case number of bits read, the average number of bits read, and/or the space efficiency of a quasi-Gray code?

Our Recursive Partition Gray Code does provide a counter-example to any efforts to show a best-case bound of more than $\Omega(\log d)$, and our hope is that this work will contribute to a better understanding of the problem, and eventually, a tighter lower bound in the case of generating a space-optimal Gray code. 


\section{Bibliography}

[BER76] James R. Bitner, Gideon Ehrlich, and Edward M. Reingold. Efficient generation of the binary reflected gray code and its applications. Commun. ACM, 19(9):517-521, 1976.

[Bro09] Gerth Stølting Brodal. personal communication, 2009.

[Fre78] Michael L. Fredman. Observations on the complexity of generating quasigray codes. Siam Journal of Computing, 7(2):134-146, 1978.

[Gra53] Frank Gray. Pulse code communications. U.S. Patent 2632058, 1953.

[Knu05] Donald E. Knuth. The Art of Computer Programming: Generating All Tuples and Permutations, volume 4, fascicle 2. Pearson Education, Inc., Upple Saddle River, NJ, USA, 2005.

[Lei92] F. Thomson Leighton. Introduction to Parallel Algorithms and Architectures: Array, Trees, Hypercubes. Morgan Kaufmann Publishers Inc., San Francisco, CA, USA, 1992.

[MP69] Marvin Minsky and Seymour Papert. Perceptrons. MIT Press, Cambridge, Mass., 1969.

[RM08] M. Ziaur Rahman and J. Ian Munro. Integer representation and counting in the bit probe model. Algorithmica, December 2008.

[Rus01] Frank Ruskey. Combinatorial Generation. Working version of book in progress, 1995-2001. 
[Sav97] Carla Savage. A survey of combinatorial Gray codes. SIAM Review, $39(4): 605-629,1997$.

[SFMS97] Gudmund Skovbjerg Frandsen, Peter Bro Miltersen, and Sven Skyum. Dynamic word problems. J. ACM, 44(2):257-271, 1997. 